\title{
Some Analyticity Properties Arising from Asymptotic Completeness in Quantum Field Theory
}

\author{
H. Epstein ${ }^{1}$, V. Glaser ${ }^{2}$, and D. Iagolnitzer ${ }^{3}$ \\ 1 Institut des Hautes Etudes Scientifiques, F-91440 Bures sur Yvette, France \\ 2 Theoretical Division, CERN, CH-1211 Geneva, 23, Switzerland \\ 3 Service de Physique Théorique CEN SACLAY BP2, F-91190 Gif-sur-Yvette, France
}

\begin{abstract}
It is well-known that two-particle unitarity of the S-Matrix in quantum field theory implies (modulo regularity assumptions) second sheet analyticity for the 2-body scattering amplitude. Here this is first used to prove off-mass-shell analyticity for the 4 point function in a complex neighborhood of any real mass-shell point under the 3-particle threshold. Then this is applied to the study of the 5 and 6 -point functions near the real mass-shell of $2 \rightarrow 3$ and $3 \rightarrow 3$ processes below the 4-particle threshold : the results are those suggested by perturbation theory apart from the 3-particle cut and away from some submanifolds. The advantage of this method, which could presumably be extended to the exploitation of $n$-particle unitarity, is that the regularity assumptions only refer to the physical scattering amplitudes.
\end{abstract}

\section{Contents}

Introduction . . . . . . . . . . . . . . . . . . . . . . . . . . . . . . . . . . . 99

1. Basic Assumptions and the Four-Point Function . . . . . . . . . . . . . . . . . . 101

2. The Five-Point Function . . . . . . . . . . . . . . . . . . . . . . . . . . . . . 109

2.1. Notations and Completeness Equations . . . . . . . . . . . . . . . . . . . . . 109

2.2. Results . . . . . . . . . . . . . . . . . . . . . . . . . 111

3. The Six-Point Function . . . . . . . . . . . . . . . . . . . . . . . . . . . . . 116

3.1. Notations and Completeness Equations . . . . . . . . . . . . . . . . . . . . . 116

3.2. Triangle Graphs . . . . . . . . . . . . . . . . . . . . . . . . . . . . . . . 119

3.3. Results . . . . . . . . . . . . . . . . . . . . . . . . . 121

Appendix . . . . . . . . . . . . . . . . . . . . . . . . . . . 122

References . . . . . . . . . . . . . . . . . . . . . . . . . 125

\section{Introduction}

The primary source of analyticity of Green functions in quantum field theory consists of the geometric properties arising from locality and the spectral condition. However it has been known for a very long time [1-3] that the assumption of asymptotic completeness entails a considerable improvement of these analyticity 
properties such as typically second-sheet analyticity across the elastic cut for the four-point amplitude. A powerful method to obtain more general results of this type (as well as general properties in field theory) initially proposed by Symanzik [4] and extensively developed by Bros and coworkers [5], is based on the study of $n$-particle-irreducible kernels and associated Bethe-Salpeter equations. In this paper we propose a different method: first the unitarity of the $S$-matrix is exploited (in the standard way) to obtain a meromorphic continuation into the unphysical sheets; then this information is used to enlarge the domain of analyticity of Green functions off the mass-shell, obtaining in particular new analyticity on the physical sheet, with poles at the zeros of the $S$-matrix determinant, and the process is in principle to be iterated. The advantage of this method is that it requires only those extra assumptions on the physical $S$-matrix which are necessary to avoid the pathologies discovered by Martin [6]. Such pathologies are probably not excluded by the usual axioms, and explicit examples can presumably be constructed in two dimensions. Hence these assumptions seem minimal. In this paper only elastic 2-body unitarity is used, and applied to the 4, 5, and 6-point functions. For the 4-point function the second-sheet analyticity across the elastic cut, already known on the mass-shell (see $[1,2,6]$ ), is extended off the mass shell. This result coincides with that of $[5 a, 5 b]$ which was obtained with somewhat stronger assumptions in the program using irreducible kernels. For the 5 and 6-point functions, local results (described in more detail below) are then obtained at real points near the mass shell below the 4-particle threshold in the total center of mass energy. Apart from the 3-particle cut (whose crossing would require exploiting the 3-particle unitarity), and certain parasitic submanifolds (whose removal would require a more detailed treatment of the 2-body threshold) the results are those suggested by perturbation theory and, on mass-shell, equivalent to the properties of macrocausality and macrocausal factorization of the $S$-matrix [7]. They agree with those of Bros [5d, 5e], although the technical limitations appearing in the two approaches are somewhat different. Similar results are easily obtained for the 3-point function. This strongly suggests that the corresponding assumptions for the $S$-matrix in all possible channels imply that the analytic structure of the Green functions near real points is just as indicated by perturbation theory. It is to be noted that the technique of obtaining analyticity from unitarity is closely related to that of many works in $S$-matrix theory (see [8-13] and references therein).

We consider a theory of one scalar neutral field satisfying besides the usual Wightman axioms, asymptotic completeness with only one kind of particles with mass $\mu>0$ and spin 0 , and additional regularity conditions described in detail in Sect. 1 for the 4-particle $S$-matrix.

In Sect. 2 we prove the following result:

Let $P=\left(P_{1}, \ldots, P_{5}\right), P_{1}+\ldots+P_{5}=0$, be a real point on the 5-point mass-shell $P_{1}, P_{2}, P_{3} \in V^{+}, P_{4}, P_{5} \in V^{-}$satisfying:

$9 \mu^{2}<\left(P_{1}+P_{2}+P_{3}\right)^{2}<16 \mu^{2},\left(P_{i}+P_{j}-P_{k}\right)^{2} \neq \mu^{2}$ for any permutation $i, j, k$ of $1,2,3$. Then the 5-point Green function is analytic, near $P$ in complex directions satisfying $\operatorname{Im} s>0\left(s=\left(p_{1}+p_{2}+p_{3}\right)^{2}\right)$.

In Sect. 3 the 6-point Green function is studied near the real mass-shell $\left\{\left(p_{1}, \ldots, p_{6}\right) ; p_{1}+\ldots+p_{6}=0 p_{j}^{2}=\mu^{2}, j=1, \ldots, 6, p_{1}, p_{2}, p_{3} \in V^{+}, p_{4}, p_{5}, p_{6} \in V^{-}\right\}$. 
It is expressed as a sum of pole terms, (generalized) triangle graphs and a remainder. Near any $P$ and the mass-shell satisfying $9 \mu^{2}<\left(P_{1}+P_{2}+P_{3}\right)^{2}<16 \mu^{2}$, $\left(P_{i}+P_{j}-P_{k}\right)^{2} \neq \mu^{2}$ for any permutation $(i, j, k)$ of $(1,2,3)$ or $(4,5,6)$, the remainder term is analytic in complex directions satisfying $\operatorname{Im} s>0, s=\left(p_{1}+p_{2}+p_{3}\right)^{2}$.

It is expected, but not proved here, that, as a consequence of 3-particle unitarity together with the required additional regularity assumptions, the remainder term is, in fact, analytic in a complex neighborhood of $P$ and that the result can be extended to the points of the manifolds $\left(p_{i}+p_{j}-p_{k}\right)^{2}=\mu^{2}$ (at least away from two-particle thesholds). The same remark applies for the 5-point function in the above discussion.

\section{Basic Assumptions and the Four-Point Function}

We consider a theory of one scalar neutral local field $A(x)$ operating in a Hilbert space $\mathscr{H}$ and obeying the Wightman axioms including uniqueness of the vacuum $\Omega$. The spectrum of the energy momentum is assumed to be

$$
\{0\} \cup\left\{p \in V^{+} ; p^{2}=\mu^{2}\right\} \cup\left\{p \in V^{+} ; p^{2} \geqq 4 \mu^{2}\right\} .
$$

Here $\mu>0$ and the restriction of the representation of the Poincare group to states with mass $\mu$ is assumed to be irreducible with spin 0 , i.e. the theory has one kind of particles with mass $\mu$ and spin 0 . The Haag-Ruelle theory (see e.g. [14]) then defines two isometries $V_{\text {in }}$ and $V_{\text {out }}$ of the Fock space $\mathscr{F}$ into $\mathscr{H}$ and we assume the completeness of asymptotic states:

$$
\mathscr{H}_{\text {in }} \equiv V_{\text {in }} \mathscr{F}=\mathscr{H}=\mathscr{H}_{\text {out }} \equiv V_{\text {out }} \mathscr{F} .
$$

A further essential assumption of a dynamical nature (continuity of the 2-body $S$-matrix kernel) will be formulated later.

As a more technical assumption, not indispensable but convenient for our purposes, we assume the existence of "sharp" time-ordered products as described, e.g., in [15].

\section{The Momentum Space Analytic Function}

Let $H_{n}$ denote the $n$-point momentum-space analytic function, $H_{n}^{\prime}$ its amputated version, i.e.

$$
H_{n}^{\prime}\left(k_{1}, \ldots, k_{n}\right)=\left[\prod_{j=1}^{n}\left(k_{j}^{2}-\mu^{2}\right)\right] H_{n}\left(k_{1}, \ldots, k_{n}\right)
$$

each of them being defined and holomorphic on a certain subdomain of the complex momentum space, $\left\{\left(k_{1}, \ldots, k_{n}\right) \in \mathbb{C}^{4 n} ; \sum_{j=1}^{n} k_{j}=0\right\}$. As it is well-known, various boundary values (in the sense of tempered distributions) of $H_{n}$ yield various generalized retarded functions and also the connected chronological function

$$
\begin{gathered}
\tilde{\tau}_{c}\left(p_{1}, \ldots, p_{n}\right)=\lim _{\substack{\varepsilon \rightarrow 0 \\
\varepsilon>0}} H_{n}\left((1+i \varepsilon) p_{1}^{0}, \mathbf{p}_{1}, \ldots,(1+i \varepsilon) p_{n}^{0}, \mathbf{p}_{n}\right) \\
\tilde{\tau}_{c}(p) \delta\left(\sum_{j=1}^{n} p_{j}\right)=\int\left[\exp i \sum_{j=1}^{n} p_{j} x_{j}\right]\left(\Omega, T\left(A\left(x_{1}\right) \ldots A\left(x_{n}\right)\right) \Omega\right)_{c} d x_{1} \ldots d x_{n} .
\end{gathered}
$$


The antichronological function is obtained by changing the sign of $\varepsilon$ in (3).

\section{Reduction Formulae}

For any $\Psi \in \mathscr{H},\left\langle r_{1}, \ldots, r_{m}\right.$, in $\left.| \Psi\right)$ is the value at $\left(r_{1}, \ldots, r_{m}\right)$ of the wave-function of the $m$-particle component in $\mathscr{F}$ of $V_{\text {in }}^{*} \Psi$. Hence it is a symmetric $L^{2}$ function of $\left(r_{1}, \ldots, r_{m}\right)$ on the real manifold

$$
\left\{\left(r_{1}, \ldots, r_{m}\right): \forall j, \quad r_{j} \in V^{+} \quad \text { and } \quad r_{j}^{2}=\mu^{2}\right\}
$$

equipped with the measure

$$
\prod_{j=1}^{m} \delta\left(k_{j}^{2}-\mu^{2}\right) \theta\left(k_{j}^{0}\right) d^{4} k_{j} .
$$

Suppose, in particular, that $\Psi=\mathscr{O}(X) \Omega$, where $X=(1, \ldots, n)$ and $\mathcal{O}(X)$ is a finite linear combination of terms of the form

$$
\int\left(T\left(X_{1}\right) \ldots T\left(X_{\gamma}\right) \varphi\left(x_{1}, \ldots, x_{n}\right) d x_{1} \ldots d x_{n},\right.
$$

$\left(X_{1}, \ldots, X_{\gamma}\right.$ being some partition of $\left.X, \varphi \in \mathscr{S}\left(\mathbb{R}^{4 n}\right)\right)$. Then, in the sense of distributions on the manifold (5), wherever $r_{j} \neq r_{k}$ for all $j \neq k,\left\langle r_{1}, \ldots, r_{m}\right.$ in $\left.| \mathcal{O}(X) \Omega\right)$ coincides with the restriction to the manifold (5) of the tempered distribution

$$
\left[\prod_{j=1}^{m}\left(r_{j}^{2}-\mu^{2}\right)\right] \int\left(\exp i \sum_{j=1}^{m} r_{j} y_{j}\right)\left(\Omega, y_{1} \downarrow \ldots y_{m} \downarrow \mathcal{O}(X) \Omega\right) d y_{1} \ldots d y_{m}
$$

abbreviated as

$$
\left[\prod_{j=1}^{m}\left(r_{j}^{2}-\mu^{2}\right)\right]\left(\Omega, \tilde{r}_{1} \downarrow \ldots \downarrow \tilde{r}_{m} \downarrow \mathcal{O}(X) \Omega\right) .
$$

It is a well-known fact that the tempered distribution (9) is restrictible to the manifold (5) at non-colinear points. In the case $m=2$ it is possible to prove that (9) is restrictible to the manifold (5) everywhere and that it everywhere coincides with $\left\langle r_{1}, r_{2}\right.$, in $\left.| \mathcal{O}(X) \Omega\right)$ in the sense of tempered distributions on (5). Similarly, at non-colinear points,

$$
\left(\Omega, \mathcal{O}(X) \mid r_{1}, \ldots, r_{m}, \text { in }\right\rangle=\left[\prod_{j=1}^{m}\left(r_{j}^{2}-\mu^{2}\right)\right]\left(\Omega,\left(-\tilde{r}_{1}\right) \downarrow \ldots \downarrow\left(-\tilde{r}_{m}\right) \downarrow \mathcal{O}(X) \Omega\right)
$$

(with a similar improvement in the case of 2-particle states) and

$$
\left\langle r_{1}, \ldots, r_{m} \operatorname{out} \mid \mathcal{O}(X) \Omega\right\rangle=\left[\prod_{j=1}^{m}\left(r_{j}^{2}-\mu^{2}\right)\right]\left(\Omega, \tilde{r}_{1} \uparrow \ldots \uparrow \tilde{r}_{m} \uparrow \mathcal{O}(X) \Omega\right)
$$

etc..... An interesting special case is

$$
\mathcal{O}(X)=\int R_{\mathscr{S}}(X)\left[\exp i \sum_{j=1}^{n} k_{j} x_{j}\right] \varphi\left(\sum_{j} x_{j}\right) d x_{1} \ldots d x_{n}, \quad \varphi \in \mathscr{S}\left(\mathbb{R}^{4}\right),
$$

where $R_{\mathscr{S}}(X)$ is the generalized retarded operator corresponding to the cell $\mathscr{S}$ and $k_{j}=p_{j}+i q_{j},(1 \leqq j \leqq n), q$ in the cone corresponding to $\mathscr{S},\left(\sum_{j=1}^{n} q_{j}=0\right)$. 
Finally we recall that, if $r_{1}, \ldots, r_{m}$ (resp. $\left.r_{1}^{\prime}, \ldots, r_{\ell}^{\prime}\right)$ are non-colinear, the amputated truncated chronological function

$$
\prod_{j=1}^{m}\left(r_{j}^{2}-\mu^{2}\right) \prod_{k=1}^{\ell}\left(r_{k}^{\prime 2}-\mu^{2}\right) \tilde{\tau}_{c}\left(r_{1}, \ldots, r_{m},-r_{1}^{\prime}, \ldots,-r_{\ell}^{\prime}\right)
$$

is restrictible to the "mass-shell" manifold

$$
\left\{r, r^{\prime} ; \sum_{j=1}^{m} r_{j}-\sum_{k=1}^{\ell} r_{k}^{\prime}=0, \quad \begin{array}{r}
r_{j} \in V^{+}, r_{j}^{2}=\mu^{2}, 1 \leqq j \leqq m, \\
\left.r_{k}^{\prime} \in V^{+}, r_{k}^{\prime 2}=\mu^{2}, 1 \leqq k \leqq \ell\right\},
\end{array}\right.
$$

where it coïncides, in the sense of tempered distributions, with the kernel of the connected $S$-matrix

$$
\left.\left\langle r_{1}, \ldots, r_{m} \text { out }\right| r_{1}^{\prime}, \ldots, r_{\ell}^{\prime} \text { in }\right\rangle_{c} .
$$

References for the above mentioned facts are [16-18].

\section{Expression of Asymptotic Completeness}

Asymptotic completeness is equivalent to the set of all equations of the form

$$
\begin{aligned}
&(\Omega, \mathcal{O}(X) \mathcal{O}(Z) \Omega)=\sum_{m=0}^{\infty} \int\left(\Omega, \mathcal{O}(X) \mid k_{1}, \ldots, k_{m} \text { in (out) }\right\rangle \\
&\left.\cdot\left\langle k_{1}, \ldots, k_{m} \text { in (out) }\right| \mathcal{O}(Z) \Omega\right) \prod_{j=1}^{m} \delta\left(k_{j}^{2}-\mu^{2}\right) \theta\left(k_{j}^{0}\right) d^{4} k_{j} .
\end{aligned}
$$

These equations can be transformed into equations involving the Green functions of the theory by re-expressing the integrand with the help of the reduction formulae recalled above: this was done in [19]. In this paper the difficulty that reduction formulae may not hold at colinear points will not arise because only two-particle asymptotic states will occur as intermediate states. More precisely we shall use

$$
\begin{aligned}
& \int\left(\Omega, \tilde{T}\left(p_{1}, \ldots, p_{r}\right) \tilde{T}\left(p_{r+2}, \ldots, p_{n}\right) \Omega\right) \cdot \varphi\left(p_{1}, \ldots, p_{r}\right) \cdot \psi\left(p_{r+1}, \ldots, p_{n}\right) d p_{2} \ldots d p_{n} \\
& =\int\left[\int\left(\Omega,\left(-\tilde{k}_{1}\right) \downarrow\left(-\tilde{k}_{2}\right) \downarrow \tilde{T}\left(p_{1}, \ldots, p_{r}\right) \Omega\right) \varphi\left(p_{1}, \ldots, p_{r}\right) d p_{1} \ldots d p_{r}\right] \\
& \quad \cdot\left[\int\left(\Omega,\left(\tilde{k}_{1}\right) \downarrow\left(\tilde{k}_{2}\right) \downarrow \tilde{T}\left(p_{r+1}, \ldots, p_{n}\right) \Omega\right) \psi\left(p_{r+1}, \ldots, p_{n}\right) d p_{r+1} \ldots d p_{n}\right] \\
& \quad \cdot \prod_{j=1,2} \delta\left(k_{j}^{2}-\mu^{2}\right) \theta\left(k_{j}^{0}\right) d^{4} k_{j}
\end{aligned}
$$

provided $\varphi, \psi \in \mathscr{S}$ with

$$
\operatorname{Supp} \varphi \subset\left\{\left(p_{1}, \ldots, p_{r}\right) ; 0<\left(p_{1}+\ldots+p_{n}\right)^{2}<9 \mu^{2}\right\}
$$

and

$$
\operatorname{Supp} \psi \subset\left\{\left(p_{r+1}, \ldots, p_{n}\right) ; 0<\left(p_{r+1}+\ldots+p_{n}\right)^{2}<9 \mu^{2}\right\} .
$$

The same relation holds with $\downarrow$ replaced by $\uparrow$. Note that the meaning of (13) is that each square bracket in the integrand is restrictible to the 2-particle mass-shell manifold, where it defines an $L^{2}$-function; the product is defined as the product of these $L^{2}$-functions. 


\section{The Four-Point Function}

The domain of analyticity of $H_{4}^{\prime}$ is known [20] to contain a set of the form

$$
\mathscr{N} \cap\left\{k ;\left(k_{1}+k_{2}\right)^{2} \notin 4 \mu^{2}+\mathbb{R}^{+}\right\},
$$

where $\mathcal{N}$ is an open complex domain containing the real set

$$
\begin{aligned}
\mathscr{R}=\{ & \left(k_{1}, \ldots, k_{4}\right) \in \mathbb{R}^{16} ; \quad \sum_{j=1}^{4} k_{j}=0, \quad k_{1} \in V^{+}, k_{2} \in V^{+}, \\
& k_{3} \in V^{-}, k_{4} \in V^{-}, \quad k_{j}^{2}<4 \mu^{2}, \quad j=1,2,3,4, \\
& \left.\left(k_{1}+k_{3}\right)^{2}<\mu^{2},\left(k_{1}+k_{4}\right)^{2}<\mu^{2}\right\} .
\end{aligned}
$$

Since the holomorphy domain of $H_{4}^{\prime}$ is invariant under the complex Lorentz group, $\mathscr{N}$ can be chosen to be invariant under $L(\mathbb{C})$. It is also invariant under the symmetries $\left(k_{1} \leftrightarrow k_{2}\right)$ and $\left(k_{3} \leftrightarrow k_{4}\right)$.

$\mathscr{R}$ contains the real subset

$$
\begin{array}{r}
\mathscr{E}=\left\{\left(k_{1}, \ldots, k_{4}\right) \in \mathbb{R}^{16} ; \quad \sum_{j=1}^{4} k_{j}=0, \quad k_{1}, k_{2} \in V^{+},\right. \\
\left.k_{3}, k_{4} \in V^{-}, \quad m_{1}^{2} \leqq k_{j}^{2} \leqq m_{2}^{2}, \quad j=1,2,3,4\right\},
\end{array}
$$

where $0<m_{1}<\mu<m_{2}, m_{2}-m_{1}<\mu$, (e.g. $m_{1}=\frac{3}{4} \mu, m_{2}=\frac{3}{2} \mu$ ). In the variables

$$
\begin{aligned}
& \sigma=k_{1}+k_{2} \quad s=\sigma^{2} \\
& p=\frac{1}{2}\left(k_{1}-k_{2}\right) \\
& q=\frac{1}{2}\left(k_{3}-k_{4}\right)
\end{aligned}
$$

the set $\mathscr{E}$ takes the form

$$
\begin{aligned}
\mathscr{E}= & \left\{(\sigma, p, q) \in \mathbb{R}^{12} ; \quad \frac{\sigma}{2} \pm p \in V^{+}, \frac{\sigma}{2} \pm q \in V^{+},\right. \\
& \left.m_{1}^{2} \leqq\left(\frac{\sigma}{2} \pm p\right)^{2} \leqq m_{2}^{2}, m_{1}^{2} \leqq\left(\frac{\sigma}{2} \pm q\right)^{2} \leqq m_{2}^{2}\right\}
\end{aligned}
$$

Let $u=(1, \mathbf{0}) \in \mathbb{R}^{4}, \mathscr{V}_{u}=\left\{(\sigma, p, q) \in \mathbb{C}^{12} ; \sigma=\sqrt{s} u\right\}=\left\{(\sigma, p, q) \in \mathbb{C}^{12} ; \boldsymbol{\sigma}=0\right\}$. Then

$$
\begin{aligned}
\mathscr{E} \cap \mathscr{V}_{u}= & \underset{\sqrt{s \in 2 m_{1}+\mathbb{R}^{+}}}{\bigcup_{1}}\{\sigma=(\sqrt{s}, \mathbf{0})\} \times\left\{p ; \frac{\sigma}{2} \pm p \in V^{+}, m_{1}^{2} \leqq\left(\frac{\sigma}{2} \pm p\right)^{2} \leqq m_{2}^{2}\right\} \\
& \cdot\left\{q ; \frac{\sigma}{2} \pm q \in V^{+}, m_{1}^{2} \leqq\left(\frac{\sigma}{2} \pm q\right)^{2} \leqq m_{2}^{2}\right\} .
\end{aligned}
$$

Simple considerations of general topology about compact sets then yield:

Lemma. For every $s_{0} \in 4 m_{1}^{2}+\mathbb{R}^{+}$, there exists $\varrho\left(\sqrt{s_{0}}\right)>0$ and a complex open set $\Omega\left(\sqrt{s_{0}}\right)$ in $\mathbb{C}^{4}$, invariant under the real orthogonal group $O(3, \mathbb{R})$ (i.e. real Lorentz 
transformations leaving $u$ invariant), such that

1) $\Omega\left(\sqrt{s_{0}}\right) \supset\left\{p \in \mathbb{R}^{4}: \sqrt{s_{0}} u \pm 2 p \in V^{+}, 4 m_{1}^{2} \leqq\left(\sqrt{s_{0}} u \pm 2 p\right)^{2} \leqq 4 m_{2}^{2}\right\}$,

2) $\mathscr{N} \supset\{(\sigma, p, q ; \sigma=z u, z \in D, p \in \Omega(\operatorname{Re} z), q \in \Omega(\operatorname{Re} z)\}$

with

$$
D=\left\{z \in \mathbb{C}, 2 m_{1}<\operatorname{Re} z,|\operatorname{Im} z|<\varrho(\operatorname{Re} z)\right\} .
$$

If $\left(k_{1}, k_{2}, k_{3}, k_{4}\right)$ is real and belongs to $\mathscr{E}$ [see (17)], the following equations hold (in the sense of tempered distributions):

$$
\begin{aligned}
&\left(\Omega, \tilde{T}\left(k_{1}, k_{2}, k_{3}, k_{4}\right) \Omega\right)_{c}^{\mathrm{amp}}=\left(\Omega, \tilde{k}_{3} \downarrow \tilde{k}_{4} \downarrow \tilde{T}\left(k_{1}, k_{2}\right) \Omega\right)_{c}^{\mathrm{amp}} \\
&=h(\sigma+i 0 u, p, q) \delta\left(\sum_{j=1}^{4} k_{j}\right), \\
&\left(\Omega, \tilde{\bar{T}}\left(k_{1}, k_{2}, k_{3}, k_{4}\right) \Omega\right)_{c}^{\mathrm{amp}}=\left(\Omega, \tilde{k}_{1} \downarrow \tilde{k}_{2} \downarrow \tilde{T}\left(k_{3}, k_{4}\right) \Omega\right)_{c}^{\mathrm{amp}} \\
&=h(\sigma-i 0 u, p, q) \delta\left(\sum_{j=1}^{4} k_{j}\right), \\
&\left(\Omega, \tilde{T}\left(k_{1}, k_{2}, k_{3}, k_{4}\right) \Omega\right)_{c}^{\mathrm{amp}}+\left(\Omega, \tilde{T}\left(k_{1}, k_{2}, k_{3}, k_{4}\right) \Omega\right)_{c}^{\mathrm{amp}} \\
&=\left(\Omega, \tilde{T}\left(k_{1}, k_{2}\right) \tilde{T}\left(k_{3}, k_{4}\right) \Omega\right)_{c}^{\mathrm{amp}}
\end{aligned}
$$

where we have denoted, for complex $\sigma, p, q$

$$
h(\sigma, p, q)=H\left(\frac{\sigma}{2}+p, \frac{\sigma}{2}-p,-\frac{\sigma}{2}+q,-\frac{\sigma}{2}-q\right)
$$

and, for real $\left(k_{1}, \ldots, k_{4}\right) \in \mathscr{E}$,

$$
h(\sigma \pm i 0 u, p, q)=\lim _{\substack{\eta \in V^{+} \\ \eta \rightarrow 0}} h(\sigma \pm i \eta, p, q) .
$$

This last limit holds in the sense of distributions. In fact, owing to the domain of analyticity described above, for every $\varphi \in \mathscr{C}^{\infty}(\mathbb{R})$ with sufficiently small support $I$, the limits

$$
\lim _{\substack{\varepsilon \rightarrow 0 \\ \varepsilon>0}} \int \varphi(t) h(\sigma+(t \pm i \varepsilon) u, p, q) d t=\int \varphi(t) h(\sigma+(t \pm i 0) u, p, q) d t
$$

exist as $\mathscr{C}^{\infty}$ functions of $\sigma, p, q$, holomorphic in $p$ and $q$ in a certain domain: if, in particular, $\sigma=z u$ with real $z>2 m_{1}, p$ and $q$ may vary independently in $\bigcap_{t \in I} \Omega(z+t)$.

The completeness Eq. (13) yields, for $\left(\frac{\sigma}{2}+p, \frac{\sigma}{2}-p,-\frac{\sigma}{2}+q,-\frac{\sigma}{2}-q\right) \in \mathscr{E}$, $4 \mu^{2} \leqq \sigma^{2} \leqq 9 \mu^{2}$,

$$
\begin{aligned}
h(\sigma+i 0 u, p, q)-h(\sigma-i 0 u, p, q)= & \int h(\sigma+i 0 u, p,-r) h(\sigma-i 0 u, r, q) \\
& \cdot \frac{1}{2} \delta(\sigma \cdot r) \delta\left(r^{2}+\frac{\sigma^{2}}{4}-\mu^{2}\right) d^{4} r
\end{aligned}
$$


The meaning of this equation is the following: according to the preceding discussion $h(\sigma+i 0 u, p,-r)$, [respectively $h(\sigma-i 0 u, r, q)]$ defines an $L^{2}$ function of $\sigma$ and $r$ on the manifold $\sigma \cdot r=0, r^{2}=-\frac{\sigma^{2}}{4}+\mu^{2}$, with the measure $\delta(\sigma \cdot r) \delta\left(r^{2}+\frac{\sigma^{2}}{4}-\mu^{2}\right) d^{4} \sigma d^{4} r$, with values in the holomorphic functions of $p$ and $q$. The integrand in the r.h.s. of (26) is to be understood as the product of these $L^{2}$ functions, and the equality of both sides holds for almost every $\sigma$. Owing to the analyticity of $h(\sigma, p, q)$ described above, it can easily be shown that if we substitute $\sigma=z u$, then, for a.e. $z$ in $[2 \mu, 3 \mu]$, the two sides of Eq. (26) define the same function of $p$ and $q$ holomorphic in $\Omega(z) \times \Omega(z)$, depending in a locally $L^{2}$ way on $z$.

\section{The Two-Body S-Matrix}

The restriction of $h(\sigma+i 0 u, p, q)$ to the real submanifold

$$
\begin{array}{ll}
\left(\frac{\sigma}{2} \pm p\right)^{2}=\mu^{2}, & \left(\frac{\sigma}{2} \pm q\right)^{2}=\mu^{2} \\
\frac{\sigma}{2} \pm p \in V^{+}, & \frac{\sigma}{2} \pm q \in V^{+}
\end{array}
$$

is the kernel of the two-body $S$-matrix. In this subsection we shall recapitulate a well-known result $[1-3,5 a, 6,10]$ according to which elastic unitarity allows this function to be analytically continued across the "s-cut" into a second sheet. This recapitulation will serve to formulate our hypotheses with precision.

Let $G_{r}$ (respectively $G$ ) denote the real (respectively complex) group of rotations (i.e. Lorentz transformations leaving $u$ invariant), let $n$ be the vector $(0,1,0,0)$, and $G_{(r)}(n)=\left\{g \in G_{(r)}: g n=n\right\}$. Let us denote

$$
K_{z}\left(R_{1}, R_{2}\right)=h\left(z u, \sqrt{\frac{z^{2}}{4}-\mu^{2}} R_{1} n,-\sqrt{\frac{z^{2}}{4}-\mu^{2}} R_{2} n\right) \frac{2 \eta}{z} \sqrt{\frac{z^{2}}{4}-\mu^{2}}
$$

(where $\sqrt{\frac{z^{2}}{4}-\mu^{2}}$ is defined to have a cut on $z^{2} \in 4 \mu^{2}+\mathbb{R}^{+}$and a positive imaginary part in the cut plane). This is an analytic function of $\left(z, R_{1}, R_{2}\right) \in \mathbb{C} \times G \times G$ in

$$
\left\{z, R_{1}, R_{2}: z \in D, z^{2} \notin 4 \mu^{2}+\mathbb{R}^{+}, R_{j} \sqrt{\frac{z^{2}}{4}-\mu^{2}} n \in \Omega(z), j=1,2\right\} .
$$

For any $S_{j} \in G(n), R_{j} \in G$ with $R_{j} \sqrt{\frac{z^{2}}{4}-\mu^{2}} n \in \Omega_{z},(j=1,2)$,

$$
K_{z}\left(R_{1}, R_{2}\right)=K_{z}\left(R_{1} S_{1}, R_{2} S_{2}\right) .
$$

According to the preceding considerations, for a.e. $z \in[2 \mu, 3 \mu], K_{z \pm i 0}\left(R_{1}, R_{2}\right)$ is a holomorphic function of $R_{1}, R_{2}$ in the domain described above and

$$
K_{z+i 0}\left(R_{1}, R_{2}\right)+K_{z-i 0}\left(R_{1}, R_{2}\right)=\int_{G_{r}} d R K_{z+i 0}\left(R_{1}, R\right) K_{z-i 0}\left(R, R_{2}\right)
$$

which can be rewritten, in case $R_{1}, R_{2} \in G_{r}$, as

$$
\left(1+K_{z+i 0}\right)\left(1+K_{z-i 0}\right)=1 \text {. }
$$


Here $K_{z}$ is the operator defined on $L^{2}\left(G_{r}\right) \cap\left\{f: f(R S)=f(R)\right.$ for all $R \in G_{r}$ and all $\left.S \in G_{r}(n)\right\}$ by

$$
\left(K_{z} f\right)(R)=\int_{G_{r}} d R^{\prime} K_{z}\left(R, R^{\prime}\right) f\left(R^{\prime}\right) .
$$

Under the hypotheses of this paper, using $\bar{T}(X)=T(X)^{*}$ and Eqs. (21) and (22) leads to

$$
K_{z-i 0}=K_{z+i 0} *
$$

and (27) just expresses the unitarity of the 2-body $S$-matrix in the elastic range.

This suggests studying $\left(1+K_{z}\right)^{-1}$ and, more generally $\left(1+K_{z \cdot L_{1}, L_{2}}\right)^{-1}$, where $K_{z, L_{1}, L_{2}}\left(R_{1}, R_{2}\right)=K_{z}\left(R_{1} L_{1}, R_{2} L_{2}\right), R_{1}, R_{2} \in G_{r}, L_{1}, L_{2} \in G$ with $\left.L_{j}\right] \sqrt{\frac{z^{2}}{4}-\mu^{2}} n \in \Omega(z)$, $z \in D, \operatorname{Im} z \neq 0$. This inverse is obtained by the Fredholm theory as:

$$
\left(1+K_{z, L_{1}, L_{2}}\right)^{-1}=1+D_{z, L_{1}, L_{2}}^{-1} N_{z, L_{1}, L_{2}},
$$

where $D_{z, L_{1}, L_{2}}=\operatorname{det}\left(1+K_{z, L_{1}, L_{2}}\right)$ and $N_{z, L_{1}, L_{2}}$ are holomorphic in $z, L_{1}, L_{2}$ in the same domain.

We now make the following further assumption:

\section{Hypothesis}

For $R_{1}, R_{2} \in G_{r}, K_{z}\left(R_{1}, R_{2}\right)$ extends to a continuous function of $z, R_{1}, R_{2}$ in

$$
\left\{z \in D, 9 \mu^{2}>\operatorname{Re} z^{2}>4 \mu^{2}, \varepsilon \operatorname{Im} z \geqq 0\right\} \times G_{r} \times G_{r}
$$

for $\varepsilon=1$ and $\varepsilon=-1$.

Physically this means that the 2-body $S$-matrix-kernel is supposed to be a continuous function of all its variables. Actually it has been shown by Martin [6], that this assumption is implied by the weaker assumption that the total crosssection is bounded in this range, and as will be seen presently, leads to analyticity of the scattering amplitude across the cut.

From this it immediately follows that $K_{z}\left(R_{1}, R_{2}\right)$ remains uniformly continuous in $R_{1}, R_{2}$ as $z$ approaches from below (or above) any closed subinterval of $] 2 \mu, 3 \mu$ [ and therefore (due to Hadamard's bounds on the Fredholm determinants) so do $D_{z, 1,1}$ and $N_{z, 1,1}\left(R_{1}, R_{2}\right)$ also. In particular $D_{z \pm i 0,11}$ is continuous in $z$ and, since $1+K_{z \pm i 0}$ is invertible for a.e. real $z \in[2 \mu, 3 \mu], D_{z+i 0,1,1}$ does not vanish in this interval. By continuity, there is a complex subdomain $\Delta$ of $D$, containing ] $4 \mu^{2}, 9 \mu^{2}\left[\right.$, such that $D_{z, 1,1}$ does not vanish for $z^{2} \in \Delta$. Of course $D_{z+i 0,1,1}$ does not coïncide with $D_{z-i 0,1,1}$. In fact we have, according to (28), for almost every $z \in] 2 \mu, 3 \mu[$ and hence in this whole interval, by continuity,

$$
D_{z+i 0,1,1}=D_{z-i 0,1,1}^{-1}=D_{z+i 0,1,1}^{*} \text {. }
$$

Moreover, since $D_{z, 1,1}^{-1}$ is holomorphic for $z^{2} \in \Delta, \operatorname{Im} z^{2}<0$ as well as $N_{z, 1,1}\left(R_{1}, R_{2}\right)$, the operator $\left(1+K_{z}\right)^{-1}$ is also holomorphic in the same domain and provides an analytic continuation of the operator valued function $\left(1+K_{z}\right)_{\mid \operatorname{Im} z>0}$. Note that our continuity assumption plays an essential role here since it guarantees that (29) holds for all $z \in] 2 \mu, 3 \mu[$. Without this assumption, the Eq. (29) would not necessarily hold in the sense of distributions, as Martin's counterexamples show. 
It is now clear that $\left(1+K_{z}\right)$, originally holomorphic for $z^{2} \in D, z^{2} \notin 4 \mu^{2}+\mathbb{R}^{+}$ admits $\left(1+K_{z}\right)^{-1}$ as a meromorphic continuation from both sides of the real interval $\left.z^{2} \in\right] 4 \mu^{2}, 9 \mu^{2}$ [, i.e. we have recovered the well-known two-sheeted structure. We now propose to extend this property in several ways. We first note that $\left(1+K_{z}\left(R_{1}, R_{2}\right)\right)$ is holomorphic for $z^{2} \in \Delta, R_{1}, R_{2} \in G_{r}$ and for $z^{2} \in \Delta, \operatorname{Im} z^{2}>0$,

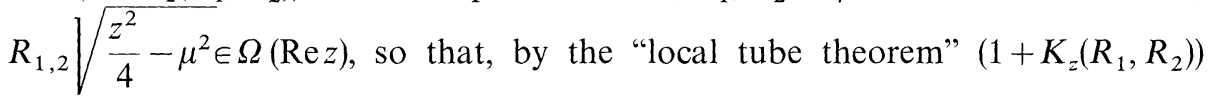
is also holomorphic in $z^{2}, R_{1}, R_{2}$ in $\left\{z^{2} \in \Delta, R_{1,2} \in V(z)\right\}, V(z)$ being some complex neighborhood of $G_{r}$ in $G$ with $G_{r} V(z)=V(z)$. In particular $K_{z \pm i 0}\left(R_{1}, R_{2}\right)$ is $\mathscr{C}^{\infty}$ in $z$, $R_{1}, R_{2}$ in $] 2 \mu, 3 \mu\left[\times G_{r} \times G_{r}\right.$, i.e. $h(\sigma \pm i 0 u, p, q)$ as a distribution has a restriction to $\left\{\sigma=z u,\left(\frac{\sigma}{2} \pm p\right)^{2}=\left(\frac{\sigma}{2} \pm q\right)^{2}=\mu^{2}, \frac{\sigma}{2} \pm p \in V^{+}, \frac{\sigma}{2} \pm q \in V^{+}\right\}$which is $\mathscr{C}^{\infty}$ for $4 \mu^{2}<z^{2}$ $<9 \mu^{2}$.

\section{Extension off the Mass-Shell}

This allows us to give a straightforward meaning to

$$
\begin{aligned}
& h((z+i 0) u, p, q)-h((z-i 0) u, p, q) \\
& \quad=\frac{1}{z} \int h((z+i 0) u, p,-r) h((z-i 0) u, r, q) \delta(u \cdot r) \delta\left(r^{2}+\frac{\sigma^{2}}{4}-\mu^{2}\right) d r
\end{aligned}
$$

at first in the case when, e.g. $p$ is real and "on the mass-shell", i.e. $u \cdot p=0, p^{2}=-\frac{z^{2}}{4}$ $+\mu^{2}, 4 \mu^{2}<z^{2}<9 \mu^{2}$. Indeed the second factor in the integrand is restrictible as a distribution on the manifold $u \cdot r=0, r^{2}=-\frac{z^{2}}{4}+\mu^{2}$ where it depends holomorphically on $q \in \Omega(z)$ [see Eq. (25)] and the first factor is $\mathscr{C}^{\infty}$ on that manifold in all its variables. Thus both sides of (30) are well-defined as distributions in $z$ depending holomorphically an $q \in \Omega(z)$ and $\mathscr{C}^{\infty}$ in $p$ (on "mass-shell"). The two sides are equal because they coïncide when integrated with test-functions in $p$ and $z$. But the r.h.s. is the continuous boundary value of a function, $\mathscr{C}^{\infty}$ in $p$, holomorphic in $z$ and $q$ in $\left\{z^{2} \in \Delta, \operatorname{Im} z^{2}<0\right\} \times \Omega(\operatorname{Re} z)$. Hence (by Schwarz's reflection principle) $h((z+i 0) u, p, q)$ extends to a function $\mathscr{C}^{\infty}$ in $p$, holomorphic in $z$ and $q$ in $\left\{z^{2} \in \Delta, q \in \Omega(\operatorname{Re} z)\right\}$. The same holds for $h((z-i 0) u, p, q)$. We now repeat this argument with both $p$ and $q$ off the "mass-shell" and thus prove:

Theorem 1. The function $h(z u, p, q)$ admits an analytic continuation across the cut $4 \mu^{2}<z^{2}<9 \mu^{2}$, from both sides to a function holomorphic in

$$
\left\{z, p, q: z^{2} \in \Delta, z^{2} \notin 4 \mu^{2}+\mathbb{R}^{+}, p, q \in \Omega(\operatorname{Re} z)\right\} .
$$

Remark. 1) It is clear that, in fact, $h(z u, p, q)$ has a meromorphic continuation, in the second sheet, in the whole of

$$
\left\{z^{2} \in D, z^{2} \notin 4 \mu^{2}+\mathbb{R}^{+}, p, q \in \Omega(\operatorname{Re} z)\right\}
$$

the only singularities being the zeroes of the (first-sheet) function $D_{z} \equiv D_{z, 1,1}$. These zeroes are resonances of the theory. It is their accumulation at the real 
interval $4 \mu^{2}<z^{2}<9 \mu^{2}$ (here prevented by our explicit assumption) which might prevent the analytic continuation of $h$ to the second sheet.

2) $D_{z}$ is entirely determined by the $S$-matrix.

3) On the mass-shell, the domain $D$ can actually be replaced by a quite sizable domain, namely the "partial-wave anakyticity" domain as obtained in $[2,21,22]$.

4) The invariance of the domain of analyticity of $h$ under the complex Lorentz group $L(\mathbb{C})$ allows an obvious extension of Theorem 1 .

\section{The Five-Point Function}

\subsection{Notations and Completeness Equations}

We denote below

$$
\begin{gathered}
T\left(p_{1}, p_{2}, p_{3}, p_{4}\right)=\left[\prod_{j=1}^{4}\left(p_{j}^{2}-\mu^{2}\right)\right] \tilde{\tau}_{c}\left(p_{1}, p_{2}, p_{3}, p_{4}\right) \delta\left(\sum_{j=1}^{4} p_{j}\right), \\
\tilde{\tau}_{c}\left(p_{1}, p_{2}, p_{3}, p_{4}\right) \delta\left(\sum_{j=1}^{4} p_{j}\right)=\int e^{i \sum_{j=1}^{4} p_{j} x_{j}} \tau_{c}\left(x_{1}, x_{2}, x_{3}, x_{4}\right) d x_{1} \ldots d x_{4},
\end{gathered}
$$

where $\tau_{c}$ is the connected chronological function. In the real region of momentum space where $0<p_{j}^{2}<4 \mu^{2},\left(p_{1}+p_{3}\right)^{2}<\mu^{2},\left(p_{1}+p_{4}\right)^{2}<\mu^{2}, 4 \mu^{2}<\left(p_{1}+p_{2}\right)^{2}<9 \mu^{2}$, $T$ is the product of $\delta\left(\sum_{j} p_{j}\right)$ by a locally analytic function $t$ (see Sect. 1). Hence its essential support at any real point $P$ in that region is the essential support of the $\delta$-function, given by

$$
E S_{P}\left(:==^{2}\right)=\left\{\left(x_{1}, x_{2}, x_{3}, x_{4}\right): x_{1}=x_{2}=x_{3}=x_{4}\right\} \text {, }
$$

where $:=2$ is a symbol for $T$.

$$
\begin{aligned}
& \text { We denote similarly: } F\left(p_{1}, \ldots, p_{5}\right)=\delta\left(\sum_{j=1}^{5} p_{j}\right) f\left(p_{1}, \ldots, p_{5}\right), \\
& f\left(p_{1}, \ldots, p_{5}\right)=\left[\prod_{j=1}^{5}\left(p_{j}^{2}-\mu^{2}\right)\right] \tilde{\tau}_{c}\left(p_{1}, \ldots, p_{5}\right),
\end{aligned}
$$

the amputated Fourier transform of the 5-point connected chronological function. The region of interest will be a real neighborhood $\mathscr{N}(\mathscr{R})$ of the region:

$$
\begin{aligned}
\mathscr{R}=\left\{\left(p_{1}, \ldots, p_{5}\right):\right. & p_{j}^{2}=\mu^{2}, j=1, \ldots, 5, p_{j} \in V^{+} \text {for } j=1,2,3, p_{j} \in V^{-} \text {for } j=4,5, \\
& \left.9 \mu^{2}<\left(p_{1}+p_{2}+p_{3}\right)^{2}<16 \mu^{2}\right\} .
\end{aligned}
$$

In this region, $f$ is the boundary value of a function (the momentum space analytic function) analytic, near the reals, in

$$
\left\{\operatorname{Im}\left(p_{4}+p_{5}\right) \in V^{-}, \operatorname{Im}\left(p_{1}+p_{2}\right) \in V^{+}, \operatorname{Im}\left(p_{1}+p_{3}\right) \in V^{+}, \operatorname{Im}\left(p_{2}+p_{3}\right) \in V^{+}\right\} .
$$

It is, as is well-known (see Sect. 1), restrictible to the (real) mass-shell region $\mathscr{R}$, (away from colinear points), and coincides there with the physical (connected) scattering amplitude $(4,5 \rightarrow 1,2,3)$. We also need other boundary values of the (amputated) momentum space analytic function in the region $\mathscr{N}(\mathscr{R})$ denoted

$$
f_{i j}^{-}\left(p_{1}, \ldots, p_{5}\right), \quad(i, j)=(1,2) \text { or }(1,3) \text { or }(2,3)
$$


with

$$
F_{i j}^{-}(p)=\delta\left(\sum_{j=1}^{5} p_{j}\right) f_{i j}^{-} .
$$

The corresponding analyticity domain is (near the reals)

$$
\left\{\operatorname{Im}\left(p_{4}+p_{5}\right) \in V^{-}, \operatorname{Im}\left(p_{i}+p_{j}\right) \in V^{-}, \operatorname{Im}\left(p_{i}+p_{k}\right) \in V^{+}, \operatorname{Im}\left(p_{j}+p_{k}\right) \in V^{+}\right\}
$$

$(i, j, k)$ being a permutation of $(1,2,3)$. In $\mathscr{N}(\mathscr{R})$,

$$
\begin{aligned}
F_{i j}^{-}(p) & =F(p)-\left[\prod_{j=1}^{5}\left(p_{j}^{2}-\mu^{2}\right)\right] \mathscr{F}(\Omega, T(i, j) T(k, 4,5) \Omega)_{c} \\
& =\left[\prod_{j=1}^{5}\left(p_{j}^{2}-\mu^{2}\right)\right] \mathscr{F}(\Omega, i \downarrow j \downarrow T(3,4,5) \Omega)_{c} .
\end{aligned}
$$

The methods of $[18,12,23]$ yield in a straightforward manner the essential supports, at any real point $P$ in $\mathscr{R}$, of $F$ and $F_{i j}^{-}$. The following result [which goes beyond (34)] is obtained:

$$
E S_{P}(F) \subset C_{1} \cup C_{2} \cup C_{3},
$$

where, for any permutation $(i, j, k)$ of $(1)-(3)$,

$$
C_{k}=\left\{\left(x_{1}, \ldots, x_{5}\right): x_{4}=x_{5}, x_{k}-x_{4} \in \bar{V}^{+}, x_{i}=x_{j}, x_{j}-x_{k} \in \bar{V}^{+}\right\}
$$

(see Fig. 1).

Fig. 1. The cone $C_{3}$

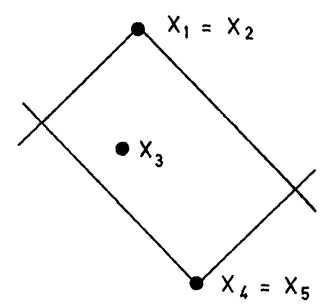

Similarly

$$
E S_{P}\left(F_{\imath j}^{-}\right) \subset C_{l} \cup C_{j} \cup C_{k}^{-},
$$

where

$$
\left.C_{k}^{-}=\left\{x_{1}, \ldots, x_{5}\right): x_{4}=x_{5}, x_{i}=x_{j}, x_{k}-x_{4} \in \bar{V}^{+}, x_{k}-x_{j} \in \bar{V}^{+}\right\} .
$$

(We recall that, so far, only microcausality and the spectrum conditions have been used.)

These essential support properties show that near any $P \in \mathscr{R}$ with $P_{i} \neq P_{j}$, both $F_{i j}^{-}$and $F$ can be restricted to the submanifold $\left\{p_{1}, \ldots, p_{5}: \sum p_{k}=0, p_{i}^{2}=\mu^{2}\right.$, $\left.p_{j}^{2}=\mu^{2}\right\}$ (where $i \neq j, i, j=1,2,3$ ). The insertion of a complete set of incoming 2 -particle states in $(34)$, for $(i, j)=(1,2)$, and the use of reduction formulae show that, in a real neighborhood of the region $\mathscr{R}$, if $p_{1} \neq p_{2}$,

$$
\begin{aligned}
F\left(p_{1}, \ldots, p_{5}\right)= & F_{1,2}^{-}\left(p_{1}, \ldots, p_{5}\right)+\int T\left(p_{1}, p_{2},-k_{1},-k_{2}\right) F_{1,2}^{-}\left(k_{1}, k_{2}, p_{3}, p_{4}, p_{5}\right) \\
& \cdot \delta\left(k_{1}^{2}-\mu^{2}\right) \theta\left(k_{1}^{0}\right) \delta\left(k_{2}^{2}-\mu^{2}\right) \theta\left(k_{2}^{0}\right) d k_{1} d k_{2}
\end{aligned}
$$


and similarly for any permutation of $1,2,3$. (For a general theory of these formulae, see e.g. $[19,5 \mathrm{~b}]$.) A graphical representation of this formula is :

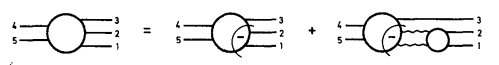

Remark. Since $t\left(p_{1}, p_{2},-k_{1},-k_{2}\right)$ has been shown to be real analytic in the integration range, and $F_{12}^{-}$is restrictible to the integration manifold, the meaning of the integral in (40) is simply that of a distribution $\left(F_{12}^{-} \delta \delta\right)$ integrated with a test-function $(t)$, the result being then multiplied by $\delta\left(\sum p_{j}\right)$.

\subsection{The Results}

Under the same assumptions as in Sect. 1, we shall prove:

Theorem 1. Let $P$ be a physical point of the process $4,5 \rightarrow 1,2,3$

$$
\left(P_{h}^{2}=\mu^{2}, k=1, \ldots, 5, \quad P_{k}^{0}>0 \text { for } k=1,2,3, \quad P_{k}^{0}<0 \text { for } k=4,5, \quad \sum_{k=1}^{5} P_{k}=0\right)
$$

such that $4 \mu^{2}<\left(P_{i}+P_{j}\right)^{2}<9 \mu^{2}$ and $\left(P_{i}+P_{j}-P_{k}\right)^{2} \neq \mu^{2}$ whenever $(i, j, k)$ is a permutation of 1)-3). The essential support of $F$ is contained in the cone $C$ :

$$
E S_{P}(F) \subset C=\left\{\left(x_{1}, \ldots, x_{5}\right): x_{4}=x_{5}, x_{1}=x_{2}=x_{3}, x_{1}-x_{4} \in \bar{V}^{+}\right\} .
$$

Equivalently, $f$ is the boundary value of a function analytic, near $P$, from directions with imaginary parts satisfying

$$
\operatorname{Im}\left(p_{1}+p_{2}+p_{3}\right) \in V^{+} .
$$

Remarks. 1) The theorem follows from the essential support properties mentioned above for $T, F, F_{i j}^{-}$and from Eq. (40).

2) The complex Lorentz invariance of the analyticity domain of $F$ combined with (43) shows that $f$ is analytic, near $P$, in directions satisfying $\operatorname{Im} s>0$, $s=\left(p_{1}+p_{2}+p_{3}\right)^{2}$. This shows that non-linear properties yield not only analyticity in "the second sheet", but also improve the analyticity in the "physical sheet".

In terms of essential supports, Lorentz invariance replaces in (42) the condition $x_{1}-x_{4} \in \bar{V}^{+}$by: $x_{1}-x_{4}=\lambda\left(P_{1}+P_{2}+P_{3}\right), \lambda \geqq 0$.

3) This analyticity shows that the restriction of $F$ to the mass-shell is itself the boundary value (from the direction $\operatorname{Im} s>0$ ) of an analytic function, near any real $P$ satisfying the hypotheses of the theorem, an appreciable improvement over the linear results of [18]. Furthermore the same method shows the same analyticity for $F_{i j}^{-}$[by inserting outgoing states in (35)] which is, therefore, also restrictible to the mass-shell.

Proof of Theorem 1. The proof of Theorem 1 is based on the general mechanism described in the introduction; however one application of this theorem, while it does yield an increase of the analyticity domain of $f$, is not sufficient to give the desired result, and two more iterations of the procedure will be needed. Since only infinitesimal results are stated in the theorem, its proof is most conveniently expressed in terms of essential supports. We shall apply general results on products 
and integrals of distributions, as stated in Theorems 10 and 11 of [23, pp. 326 and 329], to the term : $=2$ in

In particular these theorems imply that the following "rule of the game" can be applied in steps (i) to (iii) below: the only possible points $\left(x_{1}, \ldots, x_{5}\right)$ in $E S_{P}\left({ }^{\prime}={ }_{2}-O_{1}^{\prime}\right)$ are those for which there exist $\left(k_{1}, k_{2}\right)$ in the integration domain, i.e. $k_{j}^{2}=\mu^{2}, k_{j}^{0}>0, j=1,2, k_{1}+k_{2}=P_{1}+P_{2}$, and space-time points $y_{1}, y_{2}, y_{3}, y_{4}$ such that

$$
\begin{aligned}
& \left(y_{1}, y_{2}, x_{3}, x_{4}, x_{5}\right) \in E S_{\left(k_{1}, h_{2}, P_{3}, P_{4}, P_{5}\right)}(\text { ' }=\varnothing \underbrace{3}_{1}), \\
& \left(x_{1}, x_{2}, y_{3}, y_{4}\right) \in E S_{\left(P_{1}, P_{2},-k_{1},-k_{2}\right)}\left(\mathrm{O}^{2}\right) \text {, } \\
& y_{3}-y_{2}=\lambda_{1} k_{1}, \\
& y_{4}-y_{2}=\lambda_{2} k_{2} \text {, }
\end{aligned}
$$

where $\lambda_{1}, \hat{\lambda}_{2}$ are real scalars.

We shall not go into the details of the proof of this "rule of the game" which is a standard application of the theory of essential supports and is applicable whenever $P$ is not a $u=0$ point for the integral $:=0=0$ point for this integral if Eq. (44) can be satisfied with $x_{1}=x_{2}=x_{3}=x_{4}=x_{5}=0, y_{k} \neq 0$ for at least one $k$.] In particular, it is applicable in steps (i)-(iii), where $4 \mu^{2}<\left(P_{1}+P_{2}\right)^{2}$ $<9 \mu^{2}$, since the integration range does not include points where $k_{1}=k_{2}$.

In geometrical terms the rule means that $\left(x_{1}, \ldots, x_{5}\right)$ is not in $\operatorname{ES}_{P}\left({ }^{6}=\mathrm{C}_{2} \mathrm{O}_{1}^{3}\right)$ if we cannot find two space-time configurations $\left(y_{1}, y_{2}, x_{3}, x_{4}, x_{5}\right)$ and $\left(x_{1}, x_{2}\right.$, $\left.y_{3}, y_{4}\right)$ representing points in the E.S. of : $=2$, respectively, at points $\left(k_{1}, k_{2}, P_{3}, P_{4}, P_{5}\right)$ and $\left(P_{1}, P_{2},-k_{1},-k_{2}\right)$, respectively, $\left(k_{1}, k_{2}\right)$ being some point of the integration range, the two configurations being required to "fit together" in the sense that $y_{3}$ (respectively $y_{4}$ ) must lie on the space-time trajectory parallel to $k_{1}$ (respectively $k_{2}$ ) passing through $y_{1}$ (respectively $y_{2}$ ).

As already mentioned, the proof will require three steps.

\section{(i) First Step}

Let $P$ be any point in the region $\mathscr{R}$ [Eq. (33)] with $P_{1} \neq P_{2}$. From Eq. (40) it follows that $\operatorname{ES}_{P}(F)$ is contained in the intersection of $C_{1} \cup C_{2} \cup C_{3}$ (original information) with the union of the E.S. at $P$ of the two terms in the r.h.s. Since, at $P$, the E.S. of $T$ is given by Eq. (32), the "rule of the game" yields

$$
E S_{P}\left(:=\overline{2}_{1}^{3}\right) \subset C_{3}^{-} \cup C_{1,2}^{\prime},
$$

where $C_{3}^{-}$arises from the corresponding part $C_{3}^{-}$of $E S\left({ }^{5}=Q_{1}^{3}\right)$, and

$$
\begin{aligned}
C_{1,2}^{\prime}= & \left\{\left(x_{1}, \ldots, x_{5}\right): x_{1}=x_{5}, x_{1}=x_{2}, \exists\left(k_{1}, k_{2}\right) \quad\right. \text { such that } \\
& k_{1}+k_{2}=P_{1}+P_{2}, k_{1}^{2}=k_{2}^{2}=\mu^{2}, k_{1}^{0}>0, k_{2}^{0}>0, \\
& x_{3}-x_{1}=\lambda_{1} k_{1}, \exists x \quad \text { such that } \quad x-x_{1}=\lambda_{2} k_{2}, \\
& \left.x-x_{4} \in \bar{V}^{+}, x-x_{3} \in \bar{V}^{-}, \lambda_{1} \in \mathbb{R}, \lambda_{2} \in \mathbb{R}\right\} .
\end{aligned}
$$

As mentioned before, the essential support of $F$ is contained in $\left(C_{1} \cup C_{2} \cup C_{3}\right)$ $\cap\left[\left(C_{1} \cup C_{2} \cup C_{3}^{-}\right) \cup C_{12}^{\prime}\right]$, so that:

$$
E S_{P}(F) \subset C_{1} \cup C_{2} \cup C_{3}^{\prime},
$$


where

$$
\begin{aligned}
C_{3}^{\prime}= & \left\{\left(x_{1}, \ldots, x_{5}\right): x_{1}=x_{2}, x_{4}=x_{5}, x_{3}-x_{4} \in \bar{V}^{+},\right. \\
& \exists\left(k_{1}, k_{2}\right) \text { with } k_{1}^{2}=k_{2}^{2}=\mu^{2}, k_{1}^{0}>0, k_{2}^{0}>0, \\
& \left.k_{1}+k_{2}=P_{1}+P_{2}, \quad \text { such that } \quad x_{1}-x_{3}=\lambda_{1} k_{1}, \lambda_{1} \geqq 0\right\} .
\end{aligned}
$$

Here $C_{3}^{\prime}$ is bigger than $C_{1,2}^{\prime} \cap C_{3}$ and some information has thus been lost; but this will suffice for our later purposes. Configurations $\left(x_{1}, \ldots, x_{5}\right)$ occurring in (48) are schematized in Fig. $2 ; x_{3}$ must be on a trajectory parallel to $k_{1}$, passing through $x_{1}=x_{2}$, and must satisfy $x_{1}-x_{3} \in \bar{V}^{+}, x_{3}-x_{4} \in \bar{V}^{+}, x_{4}=x_{5}$.

Fig. 2. The Cone $C_{3}^{\prime}$

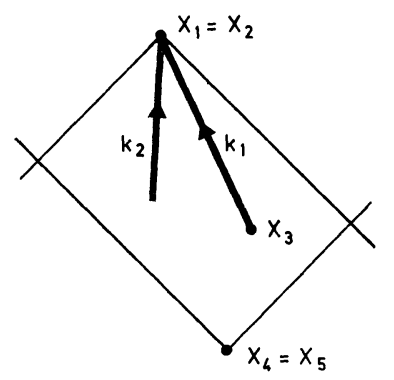

Permuting 1, 2, and 3 we obtain, for any $P$ in $\mathscr{R}$ :

$$
\begin{aligned}
E S_{P}(F) \subset C_{1}^{\prime} \cup C_{2}^{\prime} \cup C_{3}^{\prime} & \text { if } \quad P_{i} \neq P_{j}, i, j=1,2,3, i \neq j, \\
E S_{P}(F) \subset C_{i}^{\prime} \cup C_{j}^{\prime} \cup C_{k} & \text { if } \quad P_{i}=P_{j} \neq P_{h},\{i, j, k\}=\{1,2,3\} .
\end{aligned}
$$

These results, in turn, together with Eqs. (40) and (45), also yield information on $F_{i j}^{-}$:

$$
\begin{gathered}
E S_{P}\left(F_{1,2}^{-}\right) \subset C_{1}^{\prime} \cup C_{2}^{\prime} \cup C_{3}^{-} \quad \text { if } P_{i} \neq P_{j}, i, j=1,2,3, i \neq j . \\
E S_{P}\left(F_{1.2}^{-}\right) \subset C_{i}^{\prime} \cup C_{j} \cup C_{3}^{-} \quad \text { if } \quad P_{1} \neq P_{2}, P_{3}=P_{i} \quad(i=1 \text { or } 2) .
\end{gathered}
$$

\section{(ii) Second Step}

In this second step we again use Eq. (40), (i.e. first concentrate on $F_{12}^{-}$), and consider, as in the 1 . step, any real $P$ in the region $\mathscr{R}$ such that $P_{1} \neq P_{2}$. But, in the evaluation of $E S_{P}\left(:-2^{-2} \sigma^{3}\right)$, we shall now use the new information (51), (52) on $F_{12}^{-}$; Eq. (51) will be used for all $\left(k_{1}, k_{2}\right)$ in the integration domain such that $k_{1} \neq P_{3}$, $k_{2} \neq P_{3}$; Eq. (52) will be used for the points $\left(k_{1}, k_{2}\right)$ such that $k_{1}=P_{3}$ or $k_{2}=P_{3}$ if they exist.

Remark. The occurrence, in the integration domain, of points $k_{1}, k_{2}$ such that $k_{1}=P_{3}$ would be excluded if we supposed (as in Theorem 1) that $\left(P_{1}+P_{2}-P_{3}\right)^{2}$ $\neq \mu^{2}$. But this assumption cannot be made at this stage because the results of the second step will have to be reinjected into the third step.

According to the "rule of the game", a point $\left(x_{1}, \ldots, x_{5}\right)$ may be in the essential support of : $={ }_{2}^{2}$ if: $\exists k_{1}, k_{2} \in V^{+}$with $k_{1}^{2}=k_{2}^{2}=\mu^{2}, k_{1}+k_{2}=P_{1}+P_{2}, \exists y_{1} \in \mathbb{R}^{4}$, $y_{2} \in \mathbb{R}^{4}, \lambda_{1} \in \mathbb{R}, \lambda_{2} \in \mathbb{R}$ such that:

$$
x_{1}=x_{2}, x_{1}-y_{1}=\lambda_{1} k_{1}, x_{1}-y_{2}=\lambda_{2} k_{2}
$$


and

$$
\begin{array}{lll}
\left(y_{1}, y_{2}, x_{3}, x_{4}, x_{5}\right) \in C_{1}^{\prime} \cup C_{2}^{\prime} \cup C_{3}^{-} & \text {if } & k_{1} \neq P_{3} \neq k_{2} \\
\left(y_{1}, y_{2}, x_{3}, x_{4}, x_{5}\right) \in C_{1}^{\prime} \cup C_{2} \cup C_{3}^{-} & \text {if } & k_{1}=P_{3} \neq k_{2} .
\end{array}
$$

If $k_{1} \neq P_{3} \neq k_{2}$, the contribution of $C_{3}^{-}$is again in $C_{3}^{-}$and the contribution of $C_{2}^{\prime}$ or $C_{1}^{\prime}$ is contained in the set of points such that, for some $\lambda_{1}^{\prime} \in \mathbb{R}^{+}, k_{1}^{\prime} \in V^{+}, k_{2}^{\prime} \in V^{+}$, with $k_{1}^{\prime}+k_{2}^{\prime}=k_{1}+P_{3}, k_{1}^{\prime 2}=k_{2}^{\prime 2}=\mu^{2}$,

$x_{1}=x_{2}, x_{1}-x_{3}=\lambda_{1} k_{1}, x_{1}-y_{2}=\lambda_{2} k_{2}, x_{3}-y_{2}=\lambda_{1}^{\prime} k_{1}^{\prime} \in \bar{V}_{+}, y_{2}-x_{4} \in \bar{V}_{4}^{+}, x_{4}=x_{5}$.

In the case $k_{1}=P_{3}$, the contribution of $C_{3}^{-}$is in $C_{3}^{-}$, the contribution of $C_{1}^{\prime}$ and $C_{2}$ are contained in

$$
\left\{\left(x_{1}, \ldots, x_{5}\right), x_{1}=x_{2}, x_{4}=x_{5}, x_{1}-x_{3}=\lambda P_{3}, x_{3}-x_{4} \in \bar{V}^{+}\right\} .
$$

[This situation occurs only if $\left(P_{1}+P_{2}-P_{3}\right)^{2}=\mu^{2}$.]

Intersecting these sets with $C_{1} \cup C_{2} \cup C_{3}$ gives [with again some loss of information in (54)]:

$$
E S_{P}(F) \subset C_{1} \cup C_{2} \cup C_{3}^{\prime \prime},
$$

where $C_{3}^{\prime \prime}=\hat{C}_{3}^{\prime \prime} \cup \hat{\hat{C}}_{3}^{\prime \prime}$, the term $\hat{\hat{C}}_{3}^{\prime \prime}$ being absent if $\left(P_{1}+P_{2}-P_{3}\right)^{2} \neq \mu^{2}$, and where

$$
\begin{aligned}
& \hat{C}_{3}^{\prime \prime}=\left\{\left(x_{1}, \ldots, x_{5}\right): x_{4}=x_{5}, x_{1}=x_{2}, x_{3}-x_{4} \in \bar{V}^{+}, x_{3}-x_{1} \in \bar{V}^{-},\right. \\
& \exists k_{1}, k_{2} \text { with } k_{1}^{2}=k_{2}^{2}=\mu^{2}, k_{1}^{0}>0, k_{2}^{0}>0, k_{1}+k_{2}=P_{1}+P_{2} \text {, } \\
& \exists k_{1}^{\prime}, k_{2}^{\prime} \text { with } k_{1}^{\prime 2}=k_{2}^{\prime 2}=\mu^{2}, k_{1}^{\prime 0}>0, k_{2}^{\prime 0}>0, k_{1}^{\prime}+k_{2}^{\prime}=k_{1}+P_{3} \text {, } \\
& \exists y \in \mathbb{R}^{4} \quad \text { such that } \quad x_{1}-x_{3}=\lambda_{1} k_{1}, \lambda_{1} \geqq 0 \\
& x_{3}-y=\hat{\lambda}_{1}^{\prime} k_{1}^{\prime}, \lambda_{1}^{\prime} \geqq 0 \\
& \left.x_{1}-y=\lambda_{2} k_{2}, \lambda_{2} \geqq 0\right\} \text {, } \\
& \hat{\hat{C}}_{3}^{\prime \prime}=\left\{\left(x_{1}, \ldots, x_{5}\right): x_{4}=x_{5}, x_{1}=x_{2}, x_{3}-x_{4} \in \bar{V}^{+}, x_{1}-x_{3} \in \bar{V}^{+}\right. \text {, } \\
& \left.x_{1}-x_{3}=\lambda P_{3}(\lambda \geqq 0)\right\} \text {. }
\end{aligned}
$$

By permutations, this yields

$$
E S_{P}(F) \subset C_{1}^{\prime \prime} \cup C_{2}^{\prime \prime} \cup C_{3}^{\prime \prime} \text { if } P_{1} \neq P_{2} \neq P_{3} \neq P_{1} .
$$

Also

$$
E S_{P}\left(F_{\imath j}^{-}\right) \subset C_{i}^{\prime \prime} \cup C_{j}^{\prime \prime} \cup C_{k}^{-},(i, j, k)=(1,2,3) \quad \text { if } \quad P_{1} \neq P_{2} \neq P_{3} \neq P_{1} .
$$

(iii) Third Step

Now we assume that, for all permutations $(i, j, k)$ of $(1,2,3)$

$$
P_{i} \neq P_{j}, \quad\left(P_{i}+P_{j}-P_{k}\right)^{2} \neq \mu^{2} .
$$

This allows the application of the "rule of the game" together with (57) to the determination of $E S_{P}\left(=2 \sigma_{1}^{3}\right)$. The contribution of $C_{1}^{\prime \prime}$ or $C_{2}^{\prime \prime}$ consists of the points $\left(x_{1}, \ldots, x_{5}\right)$ such that there exist $k_{1} \in V^{+}, k_{2} \in V^{+}$, with $k_{1}+k_{2}=P_{1}+P_{2}$, $k_{1}^{2}=k_{2}^{2}=\mu^{2}, y_{1} \in \mathbb{R}^{4}, y_{2} \in \mathbb{R}^{4}, \lambda_{1} \in \mathbb{R}, \lambda_{2} \in \mathbb{R}$ such that: 
1) If $\left(k_{1}+P_{3}-k_{2}\right)^{2} \neq \mu^{2} \quad$ [note that $k_{j} \neq P_{3}$ because of the condition $\left.\left(P_{1}+P_{2}-P_{3}\right)^{2} \neq \mu^{2}\right]$ : there exist $k_{1}^{\prime} \in V^{+}, k_{2}^{\prime} \in V^{+}, k_{1}^{\prime \prime} \in V^{+}, k_{2}^{\prime \prime} \in V^{+}, z \in \mathbb{R}^{4}, \lambda_{1}^{\prime} \in \mathbb{R}^{+}$, $\lambda_{1}^{\prime \prime} \in \mathbb{R}^{+}, \lambda_{2}^{\prime} \in \mathbb{R}$, with $k_{1}^{\prime 2}=k^{\prime 2}=k_{1}^{\prime \prime 2}=k_{2}^{\prime \prime 2}=\mu^{2}, k_{1}^{\prime}+k_{2}^{\prime}=k_{1}+P_{3}, k_{1}^{\prime \prime}+k_{2}^{\prime \prime}=k_{1}^{\prime}+k_{2}$, such that

$$
\begin{gathered}
x_{4}=x_{5}, x_{1}=x_{2}, y_{1}=x_{3}, x_{1}-x_{3}=\lambda_{1} k_{1}, x_{1}-y_{2}=\lambda_{2} k_{2}, \\
x_{3}-y_{2}=\lambda_{1}^{\prime} k_{1}^{\prime} \in \bar{V}^{+}, y_{2}-z=\lambda_{1}^{\prime \prime} k_{1}^{\prime \prime} \in \bar{V}^{+}, x_{3}-z=\lambda_{2}^{\prime} k_{2}^{\prime} \in \bar{V}^{+} .
\end{gathered}
$$

If $\left(x_{1}, \ldots, x_{5}\right)$ is furthermore required to be in $C_{1} \cup C_{2} \cup C_{3}$, the conditions (58) are supplemented with: $\lambda_{1} k_{1} \in \bar{V}^{+}$. In this case we thus have the kinematical situation pictured in Fig. 3.

Fig. 3

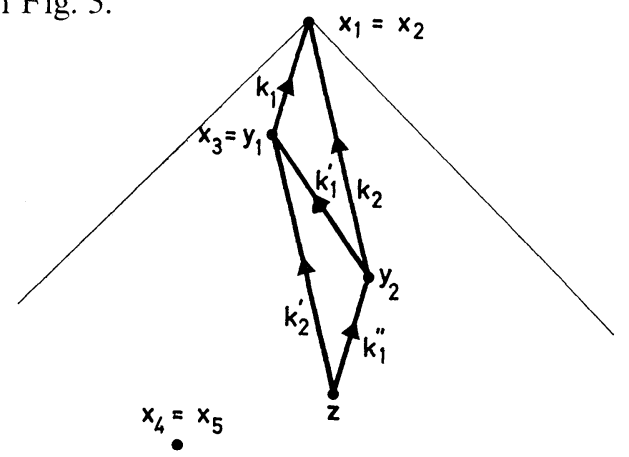

However a well-known theorem (one proof can be found e.g. in [24]) asserts that [in view of the restrictions $\left(k_{1}+k_{2}\right)^{2}=\left(P_{1}+P_{2}\right)^{2}<9 \mu^{2},\left(k_{1}^{\prime}+k_{2}^{\prime}\right)^{2}=\left(k_{1}+P_{3}\right)^{2}$ $\left.<9 \mu^{2},\left(k_{1}^{\prime \prime}+k_{2}^{\prime \prime}\right)^{2}=\left(k_{1}^{\prime}+k_{2}\right)^{2}<9 \mu^{2}\right]$ such a situation is impossible unless $\hat{\lambda}_{1}=0$, i.e.:

$$
x_{1}=x_{2}=x_{3}, x_{3}-x_{4} \in \bar{V}^{+}, x_{4}=x_{5} .
$$

2) If $\left(k_{1}+P_{3}-k_{2}\right)^{2}=\mu^{2}$, there exists $\lambda_{1}^{\prime} \geqq 0$ such that $x_{1}=x_{2}, x_{1}-x_{3}=\lambda_{1} k_{1}$, $x_{1}-y_{2}=\lambda_{2} k_{2}, x_{3}-y_{2}=\lambda_{1}^{\prime} k_{2} \in \bar{V}^{+}, y_{2}-x_{4} \subset \bar{V}^{+}, x_{4}=x_{5}$.

But this implies $\left(\lambda_{2}-\lambda_{1}^{\prime}\right) k_{2}=\lambda_{1} k_{1}$, hence, since $k_{1} \neq k_{2}, \lambda_{1}=\left(\lambda_{2}-\lambda_{1}^{\prime}\right)=0$, so that $x$ must again satisfy (59).

In conclusion, for $P \in \mathscr{R}$ with $P_{i} \neq P_{j},\left(P_{i}+P_{j}-P_{k}\right)^{2} \neq \mu^{2}$,

$$
E S_{P}(F) \subset\left\{\left(x_{1}, \ldots, x_{5}\right), x_{1}=x_{2}=x_{3}, x_{4}=x_{5}, x_{3}-x_{4} \in \bar{V}^{+}\right\} .
$$

This concludes the proof of Theorem 1 .

Remark. As it was pointed out in [18], the Steinmann identities allow a decomposition (near points of $\mathscr{R}$ ) of the various boundary values $f, f_{i j}^{-}$, of the momentum space analytic function into six functions which we denote $g_{k}^{ \pm}(k=1,2,3)$. Each $g_{h}^{ \pm}$is analytic in the intersection of a neighborhood of $\mathscr{R}$ with the tube

$$
\mathbb{R}^{16}+i \stackrel{\circ}{\tilde{C}}_{h}^{ \pm}=\left\{(p+i q): q_{j}+q_{t} \in V^{ \pm}, q_{j}+q_{k} \in V^{+}, q_{t}+q_{h} \in V^{+}\right\}
$$

$(k, \ell, j)$ being any permutation of $(1,2,3)$, and

$$
f=\sum_{k=1}^{3} g_{k}^{+}, \quad f_{\ell j}^{-}=g_{i}^{+}+g_{j}^{-}+g_{k}^{-} .
$$

It is possible to give a proof of Theorem 1 by using these functions: it turns out that each of them has the analyticity asserted for $f$ by Theorem 1 . 


\section{The Six-Point Function}

\subsection{Notations and Completeness Equations}

In this section, we consider real points $\left(P_{1}, \ldots, P_{6}\right)$ (with $\left.P_{1}+\ldots+P_{6}=0\right)$ in a real neighborhood $\mathscr{N}(\mathscr{R})$ of the set

$$
\begin{aligned}
\mathscr{R}= & \left\{\left(p_{1}, \ldots, p_{6}\right): p_{h}^{2}=\mu^{2}, k=1, \ldots, 6, p_{j} \in V^{+} \quad \text { for } j=1,2,3,\right. \\
& \left.p_{j} \in V^{-} \text {for } j=4,5,6,9 \mu^{2}<\left(p_{1}+p_{2}+p_{3}\right)^{2}<16 \mu^{2}\right\} .
\end{aligned}
$$

The condition that $\left(p_{1}+p_{2}+p_{3}\right)^{2}<16 \mu^{2}$ ensures that for any triplet $k_{1}, k_{2}, k_{3}$ with $k_{1}+k_{2}+k_{3}=p_{1}+p_{2}+p_{3}, k_{j}^{2}=\mu^{2}, k_{j}^{0}>0$ for all $j$, the inequality $\left(k_{1}+k_{2}\right)^{2}$ $<9 \mu^{2}$ holds.

We denote

$$
\begin{gathered}
F\left(p_{1}, \ldots, p_{6}\right)=\stackrel{s}{a}=E_{i}^{3}=\delta\left(\sum_{J=1}^{6} p_{j}\right) f\left(p_{1}, \ldots, p_{6}\right) \\
f(p)=\left[\prod_{j=1}^{6}\left(p_{j}^{2}-\mu^{2}\right)\right] \tilde{\tau}_{c}\left(p_{1}, \ldots p_{6}\right)
\end{gathered}
$$

the amputated Fourier transform of the 6-point connected chronological function. In a neighborhood of $\mathscr{R}, f$ is the boundary value of the 6-point momentum space analytic function from the directions verifying

$$
\left\{\operatorname{Im}\left(p_{i}+p_{j}\right) \in V^{+}, \operatorname{Im}\left(p_{t}+p_{m}\right) \in V^{-}, \operatorname{Im}\left(p_{i}+p_{J}+p_{\ell}\right) \in V^{+},\right.
$$

for every permuation $(i, j, k)$ of $(1,2,3)$ and $(/, m, n)$ of $(4,5,6)\}$.

The restriction of $f$ to the mass-shell region $\mathscr{R}$ at non-colinear points, is the connected scattering amplitude for the process $\left(-p_{4},-p_{5},-p_{6}\right) \rightarrow\left(p_{1}, p_{2}, p_{3}\right)$.

The conditions $\operatorname{Im}\left(p_{i}+p_{j}+p_{\ell}\right) \in V^{+}$in (63) are due to single particle poles. These can, as is well-known, be extracted in many ways (see e.g. [5b]) and we choose the following. Let

$$
\begin{gathered}
F_{c}(x)=\frac{i}{(2 \pi)^{4}} \int e^{-i p x}\left(p^{2}-\mu^{2}+i 0\right)^{-1}\left(\mu^{2}-L^{2}\right)^{N}\left(p^{2}-L^{2}+i 0\right)^{-N} d^{4} p \\
=\frac{\left(\mu^{2}-L^{2}\right)^{N}}{(N-1) !}\left(\frac{d}{d L^{2}}\right)^{N-1}\left(\mu^{2}-L^{2}\right)^{-1} \frac{i}{(2 \pi)^{4}} \int e^{-i p x} \\
\cdot\left[\left(p^{2}-\mu^{2}+i 0\right)^{-1}-\left(p^{2}-L^{2}+i 0\right)^{-1}\right] d^{4} p, \\
F_{c}(x)=\theta\left(x^{0}\right) F^{+}(x)+\theta\left(-x^{0}\right) F^{-}(x) \\
F^{+}(x)=\frac{\left(\mu^{2}-L^{2}\right)^{N}}{(N-1) !}\left(\frac{d}{d L^{2}}\right)^{N-1}\left(\mu^{2}-L^{2}\right)^{-1} \frac{1}{(2 \pi)^{3}} \int e^{-i p x} \\
\cdot\left[\delta\left(p^{2}-\mu^{2}\right)-\delta\left(p^{2}-L^{2}\right)\right] \theta\left(p^{0}\right) d^{4} p \\
F^{-}(x)=F^{+}(-x) .
\end{gathered}
$$

Here $L>4 \mu$, and $N>0$ is an integer so large that $F^{ \pm}, F_{c}$ are several times continuously differentiable. $F_{c}$ will serve as "propagator" in the Feynman-like diagrams we shall need. In particular we define

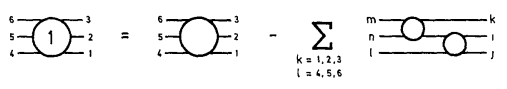


(Here the indices $i \neq j$ take the values $1,2,3, m \neq n$ take the values $4,5,6$ and there are 9 such pole terms.)

For example

$$
\begin{aligned}
: \mathrm{O}_{0}= & \int T\left(p_{2}, p_{3}, p_{6},-k\right) T\left(k, p_{1}, p_{4}, p_{5}\right) \\
& \cdot\left[\left(\mu^{2}-L^{2}\right)^{N}\left(k^{2}-\mu^{2}+i 0\right)^{-1}\left(k^{2}-L^{2}+i 0\right)^{-N}\right] d^{4} k \\
= & t\left(p_{2}, p_{3}, p_{6},-k\right) t\left(k, p_{1}, p_{4}, p_{5}\right)\left(\mu^{2}-L^{2}\right)^{N} \\
& \cdot\left(k^{2}-\mu^{2}+i 0\right)^{-1}\left(k^{2}-L^{2}+i 0\right)^{-N} \delta\left(\sum_{j=1}^{6} p_{j}\right)
\end{aligned}
$$

[in the last expression, $k$ stands for $p_{2}+p_{3}+p_{6}=-\left(p_{1}+p_{4}+p_{5}\right)$ ].

The term -1) in (65) can be written $\delta\left(\sum p_{j}\right) f_{1}(p)$, where $f_{1}$ is the boundary value of a (partially) one-particle irreducible analytic function from the directions satisfying

$$
\left\{\operatorname{Im}\left(p_{i}+p_{j}\right) \in V^{+}, \operatorname{Im}\left(p_{t}+p_{m}\right) \in V^{-},\right.
$$

for all permutations $(i, j, k)$ of $(1-3)$ and $(\ell, m, n)$ of $(4-6)\}$.

We shall also need the distributions given by

$$
\begin{aligned}
& =\mathscr{s}= \\
& =\mathscr{F}(\Omega, i \downarrow j \downarrow T(k, 4,5,6) \Omega)_{c}^{\mathrm{amp}}, \\
& =\mathscr{F}(\Omega, m \uparrow n \uparrow T(t, 1,2,3) \Omega)_{c}^{\mathrm{amp}} .
\end{aligned}
$$

In a real neighborhood of the region $\mathscr{R}$, they are equal to $\delta\left(\sum p_{j}\right)$ multiplied by boundary values of the momentum space analytic function from the directions satisfying, respectively,

$$
\begin{gathered}
\left\{\operatorname{Im}\left(p_{i}+p_{j}\right) \in V^{-}, \operatorname{Im}\left(p_{i}+p_{h}\right) \in V^{+}, \operatorname{Im}\left(p_{j}+p_{h}\right) \in V^{+}, \operatorname{Im}\left(p_{r}+p_{s}\right) \in V^{-},\right. \\
\operatorname{Im}\left(p_{r}+p_{s}+p_{t}\right) \in V^{-}, \forall(r, s) \subset(4,5,6), \quad r \neq s \\
\forall t=1,2,3\} .
\end{gathered}
$$

and

$$
\begin{gathered}
\left\{\operatorname{Im}\left(p_{m}+p_{n}\right) \in V^{+}, \operatorname{Im}\left(p_{\ell}+p_{m}\right) \in V^{-}, \operatorname{Im}\left(p_{\ell}+p_{n}\right) \in V^{-}, \operatorname{Im}\left(p_{r}+p_{s}\right) \in V^{+},\right. \\
\operatorname{Im}\left(p_{\boldsymbol{r}}+p_{s}+p_{t}\right) \in V^{+}, \forall(r, s) \subset(1,2,3), \quad r \neq s \\
\forall t=4,5,6\} .
\end{gathered}
$$

Correspondingly

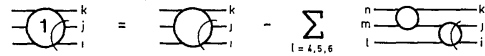

$$
\begin{aligned}
& \text { - } \sum_{1=6,5,6} i_{n=00_{1}^{k}}^{n} \\
& \text { - } \sum_{1=6,5,6} i_{n=0}^{i}
\end{aligned}
$$

with a similar identity for $\stackrel{n}{m}=-1 E_{1}^{3}$. 
The distributions 71 E and $\equiv$ are equal to $\delta\left(\sum p_{j}\right)$ multiplied by b.v. of the (partially) one particle irreducible function in domains larger than the corresponding domains (70) and (71), namely those obtained by striking out, in (70) [respectively (71)] the conditions of the type $\operatorname{Im}\left(p_{r}+p_{s}+p_{t}\right) \in V^{-}$(respectively $V^{+}$). These properties will be fully described by stating the essential supports of these distributions in the region $\mathscr{R}$.

The results of the linear program [18] give, for $P \in \mathscr{R}$,

$$
E S_{P}(\overline{Z 1})=\bigcup_{\substack{s=1,2,3 \\ t=4,5,6}} C_{\mathrm{s}}^{t}
$$

where e.g. :

$$
C_{3}^{4}=\left\{\left(x_{1}, \ldots x_{6}\right): x_{1}=x_{2}, x_{5}=x_{6}, x_{1}-x_{3} \in \bar{V}^{+}, x_{3}-x_{4} \in \bar{V}^{+}, x_{4}-x_{5} \in \bar{V}^{+}\right\}
$$

the other $C_{s}^{t}$ being obtained by independently permuting 1, 2, 3 and 4, 5, 6. Configurations occurring in $C_{3}^{4}$ are schematically represented in Fig. 4.

Fig. 4. The cone $C_{3}^{4}$

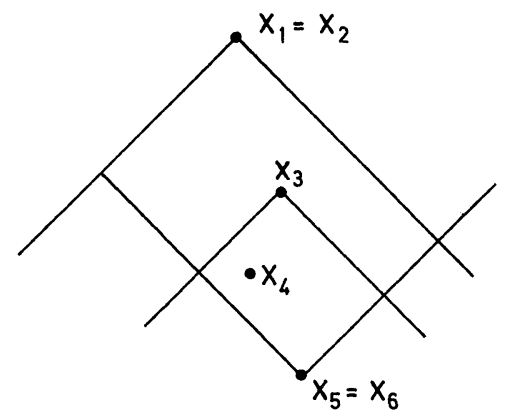

Similarly

$$
\begin{aligned}
& E S_{P}\left(\begin{array}{l}
\left.s=1 \mathcal{E}_{1}^{3}\right) \\
6
\end{array}\right) \subset \bigcup_{t=4,5,6}\left(C_{1}^{t} \cup C_{2}^{t} \cup C_{3^{-}}^{t}\right),
\end{aligned}
$$

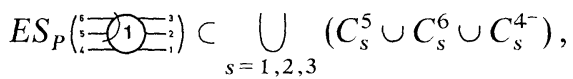

where, e.g.

$$
\begin{aligned}
C_{3^{-}}^{4}= & \left\{\left(x_{1}, \ldots, x_{6}\right): x_{1}=x_{2}, x_{5}=x_{6}, x_{1}-x_{3} \in \bar{V}^{-}, x_{3}-x_{4} \in \bar{V}^{+},\right. \\
& \left.x_{4}-x_{5} \in \bar{V}^{+}\right\} \\
C_{3}^{4^{-}=} & \left\{\left(x_{1}, \ldots, x_{6}\right): x_{1}=x_{2}, x_{5}=x_{6}, x_{1}-x_{3} \in \bar{V}^{+}, x_{3}-x_{4} \in \bar{V}^{+},\right. \\
& \left.x_{4}-x_{5} \in \bar{V}^{-}\right\} .
\end{aligned}
$$

It follows that, for any permutation $(i, j, k)$ of $(1,2,3)$ and $(\ell, m, n)$ of $(4,5,6)$, the distributions

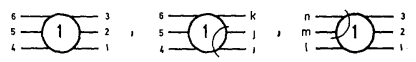

as well as their non-irreducible versions can be restricted to the manifolds $\left\{p: p_{i}^{2}=p_{j}^{2}=\mu^{2}\right\}$ and $\left\{p: p_{m}^{2}=p_{n}^{2}=\mu^{2}\right\}$ near points $P$ in $\mathscr{R}$, with $P_{i} \neq P_{j}$ or $P_{m} \neq P_{n}$ 
respectively. Our assumptions imply that the following equations [analogous to Eq. (40) of Sect. 2] are satisfied in a real neighborhood of $\mathscr{R}$ :

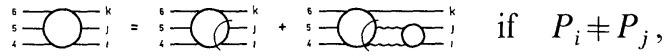

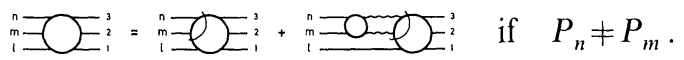

These equations make sense because of the local analyticity of the 4 point function in the integration range. Equations (77) [respectively (78)] is obtained by inserting a complete set of 2-particle ingoing (respectively outgoing) states in (the untruncated version of) Eq. (68) [respectively (69)]. Other equations of the same type can be obtained by exchanging the words "ingoing" and "outgoing". Explicitly, in the above formulae, we have, e.g.,

$$
\begin{aligned}
:=\operatorname{los}_{1}^{3}= & \int T\left(p_{1}, p_{2},-k_{1},-k_{2}\right)\left[\left(k_{1}^{2}-\mu^{2}\right)\left(k_{2}^{2}-\mu^{2}\right)\right. \\
& \cdot\left[\prod_{j=3}^{6}\left(p_{j}^{2}-\mu^{2}\right)\right] \int \exp i\left(k_{1} y_{1}+k_{2} y_{2}+p_{5} x_{3}+\ldots+p_{6} x_{6}\right) \\
& \left.\cdot\left(\Omega, y_{1} \downarrow y_{2} \downarrow T\left(x_{3}, \ldots, x_{6}\right) \Omega\right)_{c} d y_{1} d y_{2} d x_{1} \ldots d x_{6}\right] \\
& \cdot \delta\left(k_{1}^{2}-\mu^{2}\right) \theta\left(k_{1}^{0}\right) d^{4} k_{1} \delta\left(k_{2}^{2}-\mu^{2}\right) \theta\left(k_{2}^{0}\right) d^{4} k_{2} .
\end{aligned}
$$

Equations (77) and (72) yield

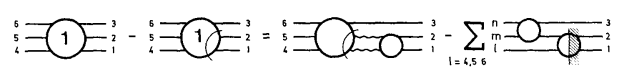

where $=$ (the discontinuity of $=\sigma$ ) satisfies

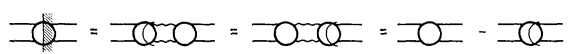

[The other pole terms in $(75)$ have no discontinuity in the channel $(1,2)$.] Similarly

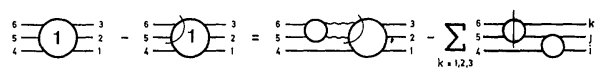

Further expansion of (79) gives

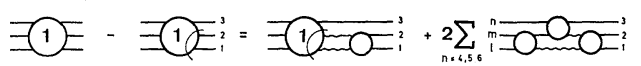

and similarly for (80).

\subsection{Triangle Graphs}

We now introduce auxiliary "six-point functions" generalizing Feynman integrals associated with triangle graphs. Their chronological boundary values, denoted $G_{k}^{\ell},(k=1,2,3, \ell=4,5,6)$ are given, in $x$-space by

$$
\begin{aligned}
G_{3}^{2}\left(x_{1}, \ldots, x_{6}\right)= & \int \tau_{c}^{\mathrm{amp}}\left(x_{1}, x_{2}, y_{1}, y_{2}\right) \tau_{c}^{\mathrm{amp}}\left(x_{3}, x_{4}, y_{3}, y_{4}\right) \tau_{c}^{\mathrm{amp}}\left(x_{5}, x_{6}, y_{5}, y_{6}\right) \\
& F_{c}\left(y_{2}-y_{3}\right) F_{c}\left(y_{4}-y_{5}\right) F_{c}\left(y_{1}-y_{6}\right) d y_{1} \ldots d y_{6} .
\end{aligned}
$$


The Fourier transform $\tilde{G}_{3}^{4}$ of $G_{3}^{4}$ in momentum-space is represented diagrammatically in Fig. 5.

Fig. 5. Diagram for $\hat{G}_{3}^{4}$

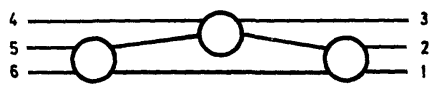

The integrand of this expression is well defined (provided $N$ has been chosen sufficiently large in the definition of $F_{c}$ ). The integration over the $y$-variables exists in the sense of a "weak adiabatic limit". This means that, if we introduce an extra factor $g_{\varepsilon}\left(y_{1}, \ldots, y_{6}\right)=g\left(\varepsilon y_{1}, \ldots, \varepsilon y_{6}\right)$ into the integrand, with $g \in \mathscr{S}\left(\mathbb{R}^{24}\right)$, $g(0)=1$, the limit exists and is independent of $g$. It defines the chronological b.v. of a 6-point-function with all the standard linear properties. These facts as well as the correct definition of the other "boundary values" and, actually a general theory of such Feynman-like diagrams can easily be derived from the ideas sketched in [15, Sect. 6]. The momentum space analytic functions so obtained coincide with the $G$-convolutions defined and studied by Bros and Lassalle by means of integrations over complex "contours". General results on analyticity of the latter in the primitive domain have been obtained in this approach in $[5,25]$. More refined results that go beyond the primitive domain, such as the derivation of the detailed analytic structure in terms of the Landau singularities which are specific of a given diagram, have also been established in certain cases, in $[5 \mathrm{e}, 26]$ and entail in particular the results below on triangular diagrams. A short alternative treatment of these triangular diagrams, sufficient for our purposes, is given for completeness in the Appendix. It yields, in particular, the following results (in momentum space)

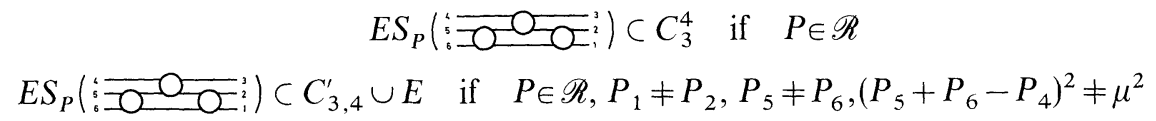

with

$$
\begin{aligned}
C_{3,4}^{\prime}= & \left\{\left(x_{1}, \ldots, x_{6}\right) ; x_{1}=x_{2}, x_{3}=x_{4}, x_{5}=x_{6}, \exists k_{1}, k_{2}, k_{3} \in V^{+},\right. \\
& k_{1}^{2}=k_{2}^{2}=k_{3}^{2}=\mu^{2}, \lambda_{1}, \lambda_{2}, \lambda_{3} \in \mathbb{R}^{+} \quad \text { such that } \\
& k_{1}+k_{2}=P_{1}+P_{2}, k_{3}+k_{2}=-\left(P_{5}+P_{6}\right), k_{1}+P_{3}=k_{3}-P_{4}, \\
& \left.x_{1}-x_{3}=\lambda_{1} k_{1}, x_{1}-x_{5}=\lambda_{2} k_{2}, x_{3}-x_{5}=\lambda_{3} k_{3}\right\} \\
& \cup\left\{\left(x_{1} \ldots x_{6}\right) ; x_{1}=x_{2}=x_{3}=x_{4}=x_{5}=x_{6}\right\}
\end{aligned}
$$

(see Fig. 6) and

$$
E=\left\{\left(x_{1}, \ldots, x_{6}\right): x_{1}=x_{2}=x_{3}, x_{4}=x_{5}=x_{6}, x_{1}-x_{4} \in \bar{V}^{+}\right\} .
$$

Remarks. 1. A more detailed analysis shows, as it also follows from [5e, 26], that in fact, for points $P$ satisfying the conditions of (84) the set $E$ can be omitted in the r.h.s. of (84), i.e. $E S_{P}\left(\tilde{G}_{3}^{4}\right) \subset C_{3,4}^{\prime}$. This is not needed for the purposes of this paper.

2. Points in $C_{3,4}^{\prime}$ (other than $x_{1}=\ldots=x_{6}$ ) are represented in Fig. 6 . The conditions on $P$ in (84) ensure that given $k_{1}, k_{2}, k_{3}$ on the mass-shell with $k_{1}+k_{2}$ $=P_{1}+P_{2}, k_{2}+k_{3}=-\left(P_{5}+P_{6}\right)$, the functions $t$ associated with each bubble are locally analytic at the respective points $\left(P_{1}, P_{2},-k_{1},-k_{2}\right),\left(k_{1}, P_{3},-k_{3}, P_{4}\right)$, 
Fig. 6. Configurations in $C_{3,4}^{\prime}$

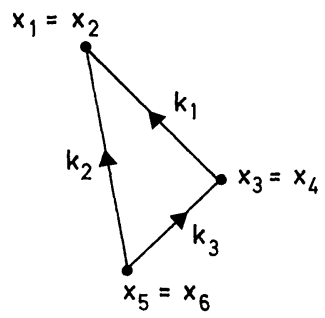

$\left(k_{2}, k_{3}, P_{5}, P_{6}\right)$. The cone $C_{3,4}^{\prime}$ then coincides with the essential support of the corresponding Feynman integral where these functions $t$ are replaced by constants.

The other result we need (see the Appendix) is

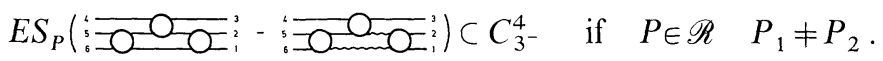

\subsection{Results}

Let $A$ be defined by

Then:

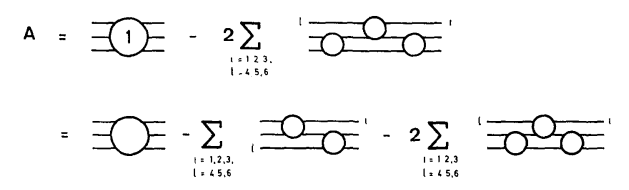

Theorem 2. At all points $P \in \mathscr{R}$ satisfying, for every permutation $(i, j, k)$ of $(1,2,3)$ or of $(4,5,6), P_{i} \neq P_{j},\left(P_{i}+P_{j}-P_{k}\right)^{2} \neq \mu^{2}$,

$$
E S_{P}(A) \subset\left\{\left(x_{1}, \ldots, x_{6}\right): x_{1}=x_{2}=x_{3}, x_{4}=x_{5}=x_{6}, x_{1}-x_{4} \in \bar{V}^{+}\right\} .
$$

Remark. The Lorentz invariance of the analyticity domain of the momentum space analytic function associated with $A$ then shows that this function is analytic, near $P$ in the directions satisfying $\operatorname{Im} s>0, s=\left(p_{1}+p_{2}+p_{3}\right)^{2}$, or, equivalently,

$$
E S_{P}(A)=\left\{\left(x_{1}, \ldots, x_{6}\right): x_{1}=x_{2}=x_{3}, x_{4}=x_{5}=x_{6}, x_{1}-x_{4}=\lambda\left(P_{1}=P_{2}+P_{3}\right), \lambda \geqq 0\right\} .
$$

Proof of Theorem 2. It will suffice to prove that, for $P \in \mathscr{R}$ satisfying the conditions of the theorem,

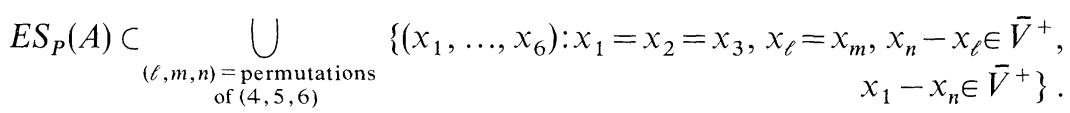

Since the same methods will obviously give a similar result with the roles of the variables $(1,2,3)$ and $(4,5,6)$ exchanged, the result $(89)$ is obtained by intersection.

By Eq. (81), for each $i=1,2,3$.

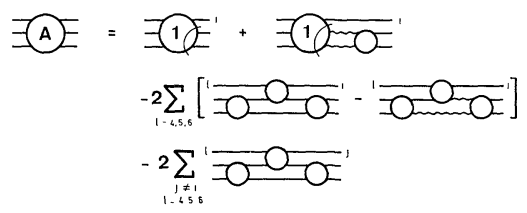


The proof of (90) is carried out in three steps completely analogous to those of Sect. 2. The cones $C_{1}, C_{2}, C_{3}$ of Sect. 2 are replaced now by

$$
C_{k}=\bigcup_{t=4,5,6} C_{k}^{t}, \quad k=1,2,3
$$

(i.e. the point $x_{4}=x_{5}$ of Sect. 2 is now replaced by

$$
\left.\bigcup_{\substack{(\ell, m, n)=\text { permutations } \\ \text { of }(4,5,6)}}\left\{\left(x_{4}, x_{5}, x_{6}\right) ; x_{n}=x_{m}, x_{\ell}-x_{n} \in \bar{V}^{+}\right\}\right) .
$$

The points $P$ considered in these three steps are such that, for every permutation $(\ell, m, n)$ of $(4,5,6), P_{\ell} \neq P_{m}$ and $\left(P_{\ell}+P_{m}-P_{n}\right)^{2} \neq \mu^{2}$. Moreover $P_{1}, P_{2}, P_{3}$ satisfy, at each step, the same conditions as in Sect. 2. In the first two steps information on $A$ follows from (91) together with (83)-(87) and yields [by (81)] information on 1 . The result (90) is the outcome of the last step. The cones $C_{i}^{\prime}, C_{i}^{\prime \prime}, C_{t}^{-}$ appearing at each step differ from those appearing in Sect. 2 in the same way as the corresponding $C_{i}$, as explained above. The supplementary terms in the r.h.s. of (91) do not modify the situation: indeed, in view of (83)-(87),

$$
\begin{array}{ll}
E S_{P}\left(\tilde{G}_{j}^{\ell}\right) \subset C_{j}, & P \in \mathscr{R} \\
E S_{P}\left(\tilde{G}_{j}^{\ell}\right) \subset C_{j}^{\prime}, & P \in \mathscr{R}, P_{i} \neq P_{k} \\
E S_{P}\left(\tilde{G}_{j}^{\ell}\right) \subset C_{j}^{\prime \prime} \quad \text { if } & P \text { satisfies the conditions of Theorem 2, }
\end{array}
$$

and the bracket term in (91) has its essential support in $C_{i}^{-}$for $P_{j} \neq P_{k}$.

\section{Appendix}

We give here a short account of the properties of triangular graphs needed in this paper, based on "discontinuity formulae" which follow straightforwardly from Sect. 6.6. of [15]. To describe diagrammatically these formulae, we shall use the following conventions:

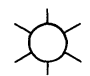

stands for the "chronological b.v." of a general $n$-point function, amputated in all its variables. It could be denoted $\langle T(X)\rangle$.

в $\{\geq$ E $\{$ A The corresponding $\langle T(A) T(B)\rangle$.

в $\left\{\bigcup_{\text {E }}\right\}$ A The corresponding $\langle T(X)-T(A) T(B)\rangle$.

An $F_{c}$ propagator.

$\tilde{y}_{2} \rightarrow y_{1} \quad F^{+}\left(y_{1}-y_{2}\right)$ or equivalently, in the present paper, $\frac{1}{(2 \pi)^{3}} \int \delta\left(k^{2}-\mu^{2}\right) \theta\left(k^{0}\right) \exp \left[-i\left(y_{1}-y_{2}\right) k\right] d^{4} k$.

Momentum space version of the preceding. 
We consider now a diagram of the form

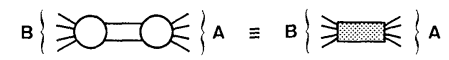

and ask for the corresponding

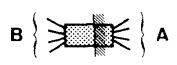

in the sense defined above. This is given, in momentum space, when $p_{A} \equiv \sum_{j \in A} p_{j}$ satisfies $p_{A} \in V^{+}, p_{A}^{2}<9 \mu^{2}$, by

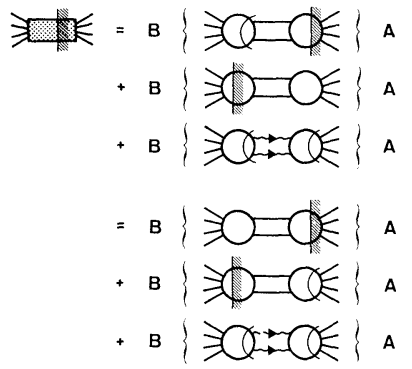

Remark. In the general case, individual terms appearing in such "discontinuity formulae" only make sense before taking the adiabatic limit (while their sum always has a well defined adiabatic limit). In the cases occurring in this paper, however, all terms make sense and the formulae hold in the adiabatic limit.

In order to apply these formulae to $\tilde{G}_{3}^{4}$, we first note that, for the 6-point function defined by a pole term,

$$
\exists E=: \overline{O O}=
$$

the $(1,2)$-channel "discontinuity"

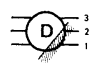

vanishes in the relevant region, i.e. at points $P$ satisfying

$$
\begin{gathered}
P_{1}+P_{2} \in V^{+},\left(P_{1}+P_{2}\right)^{2}<9 \mu^{2}, P_{j}^{2}=\mu^{2}, \quad j=3, \ldots, 6, \\
P_{3} \in V^{+}, P_{4}, P_{5}, P_{6} \in V^{-} .
\end{gathered}
$$

Hence applying (A1) yields

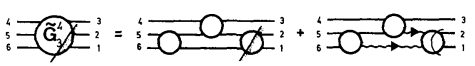

Note that, for $4 \mu^{2}<\left(P_{1}+P_{2}\right)^{2}<9 \mu^{2}$,

$$
\phi_{1}^{2}=\theta_{2}=\sigma_{m}^{2}
$$

and that (when all the variables are on the mass-shell, not at thresholds) is locally analytic. Hence the last term in (A4) is obviously well-defined, and the first is equal to

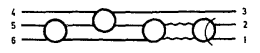

so that it is also manifestly well-defined. 
It is easy to see, by the same methods, that the momentum space analytic function associated with $\tilde{G}_{3}^{4}$ has no discontinuities (near real points $P \in \mathscr{R}$ ) in any of the channels: $\{1,3\},\{2,3\},\{4,5\},\{1,3,4\},\{2,3,4\},\{1,2,4\},\{1,2,5\},\{1,2,6\}$, $\{1,3,5\},\{1,3,6\},\{2,3,5\},\{2,3,6\}$. As a consequence, for $P \in \mathscr{R}$,

$$
E S_{P}\left(\tilde{G}_{3}^{4}\right) \subset C_{3}^{4}
$$

while the "boundary value" $\widetilde{\mathrm{G}}_{3-}^{\prime} \equiv \exists \widetilde{\mathrm{G}_{3}^{4}} \sum_{1}^{2}$ satisfies

$$
E S_{P}\left(\tilde{G}_{3^{-}}^{4}\right) \subset C_{3^{-}}^{4}
$$

According to (A4), for $P \in \mathscr{R},\left(P_{1}+P_{2}\right)^{2}>4 \mu^{2}$,

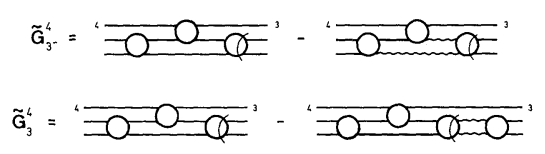

Hence

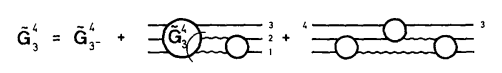

Equation (A9) implies Eq. (87) since it can be rewritten

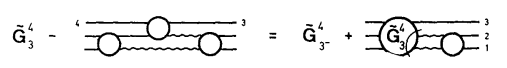

Indeed the application of the rule of the game shows that (for $P_{1} \neq P_{2}, P \in \mathscr{R}$ ) both terms in the r.h.s. have their essential support in $C_{3^{-}}^{4}$.

We now derive Eq. (84). First consider the expression

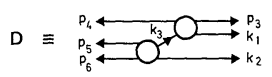

at a point $\left(k_{1}, k_{2}, p_{3}, \ldots, p_{6}\right) \in \mathscr{R}$ such that $\left(P_{5}+P_{6}\right)^{2}>4 \mu^{2},\left(P_{5}+P_{6}-P_{4}\right)^{2} \neq \mu^{2}$. These conditions imply that the bubble on the left is locally analytic, and so is the bubble on the right when $k_{3} \equiv-\left(P_{5}+P_{6}+k_{2}\right)$ is on the mass-shell, in which case

$$
\begin{array}{r}
E S_{\left(k_{1} k_{2} P_{3} \ldots P_{6}\right)}(D) \subset\left\{\left(y_{1}, y_{2}, x_{3}, \ldots, x_{6}\right) ; x_{5}=x_{6}=y_{2},\right. \\
\left.x_{4}=x_{3}=y_{1}, x_{3}-x_{5}=\varrho k_{3}, \varrho \geqq 0\right\}
\end{array}
$$

$\left(y_{1}, y_{2}\right.$ being the variables conjugate to $k_{1}, k_{2}$ ). If $k_{3}$ is not on the mass-shell then $\operatorname{ES}(D)$ is simply $\left\{x: x_{1}=x_{2}=\ldots=x_{6}\right\}$, unless $\left(k_{3}-P_{4}\right)^{2}=4 \mu^{2}$, hence $k_{1}=P_{3}$, in which case

$$
\begin{aligned}
E S_{\left(k_{1} k_{2} P_{3} \ldots P_{6}\right)}(D) \subset\left\{\left(y_{1} y_{2} x_{3}, \ldots, x_{6}\right) ; x_{5}=x_{6}=y_{2}=x_{4},\right. \\
\left.x_{3}=y_{1}, x_{3}-x_{4}=\varrho k_{1}=\varrho P_{3}, \varrho \geqq 0\right\} .
\end{aligned}
$$

Applying the "rule of the game" leads to corresponding information on "O ${ }^{2} \sigma^{3}$. The Eq. (84) is then obtained by intersecting $C_{3}^{4}$ with

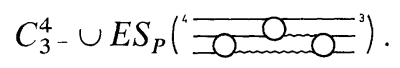

and by using arguments similar to above (and somewhat simplified) on the equation analogous to (A9) that relates $\tilde{G}_{3}^{4}$ and $\tilde{G}_{3}^{4-}$. 


\section{References}

1. Zimmermann, W.: Nuovo Cimento 21, 249 (1961)

2. Martin, A.: Scattering theory: unitarity, analyticity, and crossing. In: Lecture Notes in Physics, Vol. 3. Berlin, Heidelberg, New York: Springer 1969

3. Levy, M.: Nuovo Cimento 13, 115 (1959); Gunson, J., Taylor, J.G.: Phys. Rev. 119, 1121 (1960)

4. Symanzik, K.: J. Math. Phys. 1, 249 (1960)

5. a) Bros, J.: Analytic methods in mathematical physics, p. 85. New York: Gordon and Breach 1970

b) Bros, J., Lasalle, M.: Commun. Math. Phys. 43, 279 (1975); 54, 33 (1977)

c) Katz, A.: Thèse de 3ème cycle. Paris 1979

d) Bros, J.: Analytic structure of Green's functions in quantum field theory in mathematical problems in theoretical physics. Osterwalder, K. (ed.). In: Lecture Notes in Physics, Vol. 116, p. 166. Berlin, Heidelberg, New York: Springer 1980

e) Bros, J.: Complex analysis, microlocal calculus, and relativistic quantum theory. Iagolnitzer, D. (ed.). In: Lecture Notes in Physics, Vol. 126, p. 254, Berlin, Heidelberg, New York: Springer 1980

6. Martin, A.: Problems of theoretical physics. Moscow: Nauka 1969

7. Iagolnitzer, D., Stapp, H.P.: Commun. Math. Phys. 14, 15 (1969)

Iagolnitzer, D.: Complex analysis, microlocal calculus, and relativistic quantum theory. In: Lecture Notes in Physics, Vol. 126, p. 263. Berlin, Heidelberg, New York: Springer 1980

8. Chew, G.: The analytic $S$-matrix. New York: W. A. Benjamin 1966

9. Bloxham, M.I.W., Olive, D.I., Polkinghorne, J.C.: J. Math. Phys. 10, 494 (1969); 10, 545 (1969); 10, 553 (1969)

10. Eden, R.J., Landshoff, P.V., Olive, D.I., Polkinghorne, J.C.: The analytic S-matrix. Cambridge: Cambridge University Press 1966

11. Stapp, H.P.: Structural analysis of collision amplitudes, Balian, R., Iagolnitzer, D. (eds.), p. 191. Amsterdam: North-Holland 1976

12. Iagolnitzer, D.: The $S$-matrix. Amsterdam: North-Holland 1978

13. Iagolnitzer, D.: Commun. Math. Phys. 77, 251 (1980)

14. Jost, R.: The general theory of quantized fields. Am. Math. Soc., Providence, R.I. 1965

15. Epstein, H., Glaser, V., Stora, R.: Structural analysis of collision amplitudes. Balian, R., Iagolnitzer, D. (eds.), p. 5. Amsterdam: North-Holland 1976

16. Hepp, K.: Commun. Math. Phys. 1, 95 (1965); J. Math. Phys. 6, 1762 (1965)

Hepp, K.: Axiomatic field theory. Chrétien, Deser, S. (ed.). New York: Gordon and Breach 1966

17. Steinmann, O.: Commun. Math. Phys. 10, 245 (1968)

18. Bros, J., Epstein, H., Glaser, V.: Helv. Phys. Acta 45, 149 (1972)

19. Glaser, V., Lehmann, H., Zimmermann, W.: Nuovo Cimento 6, 1122 (1957)

20. Bros, J., Epstein, H., Glaser, V.: Nuovo Cimento 31, 1265 (1964)

21. Martin, A.: Nuovo Cimento 44A, 1219 (1966)

22. Wanders, G., Roy, S.M.: Phys. Lett. 74B, 347 (1978)

23. Iagolnitzer, D.: Structural analysis of collision amplitudes. Balian, R., Iagolnitzer, D. (eds.), p. 295. Amsterdam: North-Holland 1976

24. Iagolnitzer, D., Stapp, H.P.: Commun. Math. Phys. 57, 1 (1977)

25. Lassalle, M.: Commun. Math. Phys. 36, 185 (1974); and Thesis, Orsay 1975

26. Bros, J.: In preparation

Communicated by R. Stora

Received November 2, 1980; in revised form December 2, 1980 
